\title{
VALORACIÓN DE LOS ACTIVOS INTANGIBLES EN EL MERCADO DE CAPITALES ESPAÑOL
}

\author{
Raúl Iñiguez y Germán López
}

\author{
WP-EC 2003-04
}

Correspondencia: Germán López Espinosa, Departamento de Economía Financiera, Contabilidad y Marketing, Universidad de Alicante, Tel.: +34 965903 400, Fax: + 34965903 621, G.Lopez@ua.es.

Editor: Instituto Valenciano de Investigaciones Económicas, S.A.

Primera Edición Abril 2003

Depósito Legal: V-1866-2003

Los documentos de trabajo del IVIE ofrecen un avance de los resultados de las investigaciones económicas en curso, con objeto de generar un proceso de discusión previo a su remisión a las revistas cientificas.

\footnotetext{
* R. Iñiguez y G López: Dpto. Economía Financiera, Contabilidad y Marketing, Universidad de Alicante.
} 


\title{
VALORACIÓN DE LOS ACTIVOS INTANGIBLES EN EL MERCADO DE CAPITALES ESPAÑOL
}

\author{
Raúl Iñiguez y Germán López
}

\begin{abstract}
RESUMEN
En la actualidad existen diferencias en el contexto internacional respecto a la normativa contable aplicable a la capitalización de intangibles. En España existe una normativa estricta en cuanto al reconocimiento en el balance de los activos intangibles que posee una empresa, por ello el objetivo de este trabajo consiste en comprobar, si en nuestro mercado de capitales, el nivel de intangibles capitalizados afecta a la valoración y rentabilidad de las acciones. En este sentido, se pretende también indagar si los inversores, teniendo en cuenta la normativa contable española, perciben los activos intangibles como legítimos. Adicionalmente se analiza si el nivel de intangibles no contabilizados afecta a la rentabilidad, obteniéndose evidencia afirmativa, incluso cuando se ajusta por riesgo, cuestionándose así la validez del CAPM.
\end{abstract}

PALABRAS CLAVE: Intangibles, valoración, normativa contable, capitalización de intangibles y errores de predicción.

\begin{abstract}
In the international context there are currently important differences in the accounting rules that the firms must apply to intangible assets. In Spain the accounting for intangibles and their recognition in the balance sheet is restrictive. The objective of this study is to test if the recognized intangibles have influence on the valuation and return of the shares of the Spanish stock market. In that sense we try to investigate in the Spanish accounting regulatory framework if investors perceive intangibles to be legitimate assets. Moreover, we investigate if the level of non-recognized intangibles has influence on the returns of the shares. We confirm this hypothesis, even when we adjust by risk. This fact calls the validity of the CAPM into question.
\end{abstract}

KEY WORDS: Intangible assets, valuation, international accounting practice, capitalization, forecast errors. 


\section{Introducción}

El conocimiento, la investigación y el desarrollo, la publicidad y otras fuentes intangibles constituyen un parte de importancia creciente en las economías modernas, por lo que la contabilidad de los intangibles se ha convertido en una cuestión primordial para mejorar la calidad de la información financiera que se suministra a los usuarios de la misma.

Generalmente, los recursos empleados en activos intangibles se tratan como gasto del ejercicio en muchos sistemas contables en lugar de verse como inversiones valiosas que deben aparecer en el balance de la empresa. Capitalizar y amortizar los activos intangibles durante su vida útil produciría una mayor correlación entre los costes y beneficios futuros, lo que llevaría a una mayor riqueza informativa de los estados financieros. No obstante, dado que algunos intangibles son difíciles de registrar de forma objetiva, la relevancia de los estados financieros sería menor en un escenario en el que las empresas pudieran libremente contabilizar activos dudosos o, incluso, inexistentes. Por ello, hasta que punto se debe incrementar el reconocimiento de los intangibles es una cuestión que dependerá de la relación entre la relevancia y fiabilidad de los activos intangibles registrados.

La motivación para la realización del presente estudio proviene del debate existente sobre la utilidad de la contabilidad a la hora de recoger apropiadamente los activos intangibles, sobre todo aquellos que han sido internamente desarrollados por la empresa. Son varios los trabajos que afirman que los recientes cambios tecnológicos y económicos han hecho aumentar la importancia de los intangibles en las empresas, siendo su tratamiento contable el principal motivo de la reducción de la utilidad de los estados financieros y de la pérdida en la relevancia valorativa del resultado contable en el tiempo (véase, entre otros, Collins, Maydew y Weiss [1997], Brown, Lo y Lys [1999], Francis y Schipper [1999] y Lev y Zarowin [1999]). Si los activos intangibles afectan a esta relevancia del resultado contable es una cuestión todavía abierta, agravada además por el hecho de que la contabilidad de estos activos es muy diferente en función del país en el que estudiemos su efecto.

La justificación de esta pérdida en la relevancia de los estados financieros puede hallarse en el hecho de que la omisión de los activos intangibles en los balances debería tener un efecto debilitador de la fortaleza en la relación entre resultados y valor de la 
empresa, principalmente debido al incumplimiento del principio de correlación de gastos e ingresos cuando las inversiones en intangibles se llevan directamente a resultados. Así, tal y como indican Lev y Zarowin [1999], es en la contabilidad de los intangibles donde los sistemas contables actuales fracasan en su objetivo de reflejar el valor y la actuación de la empresa. El principal problema a resolver se centra en que estos activos son difíciles de identificar y sus beneficios futuros esperados son a menudo muy inciertos.

No obstante, también debemos indicar que para otros investigadores otros factores son más importantes, como el incremento en el porcentaje de pérdidas y partidas no recurrentes o el incremento en la volatilidad de las rentabilidades (véase Collins, Maydew y Weiss [1997] y Francis y Schipper [1999], respectivamente). A su vez, también debemos destacar que son varias las voces en contra del reconocimiento de los intangibles. Entre ellas destaca la de los analistas financieros, que consideran que la capitalización de los intangibles no mide con precisión el valor económico de dichos activos, pero sí proporciona mayores oportunidades para manipular los beneficios, haciendo más difícil su labor de predicción.

En cualquier caso, la controversia existente en la actualidad se centra en estudiar si los balances de las empresas reflejan el valor de su beneficio económico futuro. De forma general, la principal crítica que se le suele hacer a los estados financieros es su incapacidad para reflejar las diferencias en la incertidumbre de los costes y beneficios futuros de los diferentes activos que posee. De igual forma, la cuenta de resultados no refleja los diferentes grados de incertidumbre de las partidas de gastos e ingresos. Sin embargo, la mayoría de los modelos de valoración nos indican que el valor de un activo está inversamente relacionado con la incertidumbre en la corriente de futuros beneficios esperados (véase por ejemplo, Epstein y Turnbull [1980]). Este hecho es especialmente importante en el caso que estamos tratando, el de los activos intangibles, ya que éstos activos ser caracterizan por su gran incertidumbre en la cantidad y momento en que se producirá la corriente de beneficios futuros.

En este trabajo utilizamos todo tipo de información públicamente disponible en los mercados: datos contables de las empresas, datos facilitados por los analistas financieros, y datos bursátiles de sus acciones. Con esta información, se pretende analizar la influencia de los activos intangibles en el proceso de valoración de los activos financieros, tratando de investigar la relación existente entre la presencia de activos intangibles, reconocidos o no en contabilidad, y el comportamiento de las acciones en el mercado bursátil. Precisamente, una de las principales aportaciones de 
este estudio reside en que no sólo se analiza la influencia de los activos intangibles que aparecen en el balance de situación, sino que también se utiliza una aproximación de los activos intangibles que el mercado valora, pero que no tienen reflejo contable debido a la aplicación de la normativa que regula su contabilización. A su vez, si las empresas que poseen mayor proporción de activos intangibles, contabilizados o no, son percibidas como más arriesgadas, el inversor debe exigir una mayor rentabilidad. Por ello, un segundo objetivo de este trabajo será el de indagar si esto sucede en el mercado de capitales español para el período comprendido entre 1992 y 1999.

Este análisis será de especial interés tanto para los investigadores como para los organismos emisores de normas contables, puesto que permitirá establecer si la información sobre los activos intangibles disponible en los estados financieros proporciona información que es relevante para los inversores.

La estructura del trabajo se divide de la siguiente forma. A continuación nos referimos a la normativa aplicable al reconocimiento de los activos intangibles y la literatura previa, tanto nacional como internacional, que ha abordado el estudio de la relación existente entre activos intangibles y precios de mercado. En la tercera sección se explica la metodología que se va a emplear en el estudio empírico para la consecución de los objetivos del trabajo. A continuación analizamos la selección de la muestra, haciendo especial hincapié en la selección de las variables relevantes para la ejecución del análisis. En el quinto y sexto apartado se muestran los resultados del estudio, y por último, presentamos las principales conclusiones que pueden obtenerse de la evidencia presentada.

\section{Normativa y antecedentes}

Antes de entrar de lleno en la revisión de la literatura empírica que ha tratado de establecer la importancia de la información sobre los activos intangibles en la fijación de los precios de mercado de los títulos, creemos imprescindible abordar brevemente la contabilización de los intangibles según la normativa específica de los principales organismos internacionales. Esta normativa será de vital importancia para entender el entorno contable en el que se ha realizado cada uno de los trabajos empíricos que a continuación revisaremos. 


\subsection{Normativa sobre la contabilización de los intangibles}

En primer lugar, debemos precisar que se entiende por un activo intangible. Siguiendo al International Accounting Standards Committee (IASC) ${ }^{1}$ un activo intangible es un activo no monetario sin sustancia física que es mantenido para su uso en la producción o suministro de bienes o servicios, alquiler o similares, o con propósitos administrativos. A su vez, este activo es un recurso controlado por una empresa como resultado de sucesos pasados, y del que se esperan futuros beneficios económicos (véase la Norma Internacional de Contabilidad (NIC) 38, del IASC [1998]).

En cuanto a la normativa en Estados Unidos, el Financial Accounting Standards Board (FASB) indica que los activos intangibles identificables (como patentes, franquicias, marcas y similares) deben capitalizarse por su coste y amortizarse durante su vida útil estimada. Sin embargo, los activos intangibles internamente desarrollados deben contabilizarse como gasto del ejercicio. De esta forma, el coste de desarrollo, mantenimiento, y renovación de intangibles que no son específicamente identificables, tienen una vida útil indeterminada o son inherentes a la marcha continua del negocio, debe deducirse del beneficio cuando se incurren en ellos.

Dadas estas restricciones para la contabilización de los intangibles, una alternativa a considerar por los organismos emisores de normas es la de especificar un mayor abanico de condiciones bajo las cuales los costes asociados con los intangibles deberían ser capitalizados. En Estados Unidos, el FASB dio el primer paso en este sentido con la SFAS $N^{\circ} 86$, que trata la capitalización de los costes de desarrollo de software, única excepción a la SFAS $\mathrm{N}^{\circ} 2$, que establece la completa contabilización como gasto del ejercicio de los importes destinados a I+D a lo largo del ejercicio. En junio de 2001, el FASB mediante la SFAS N ${ }^{\circ} 142$ da un paso de gigante al permitir que tanto el fondo de comercio como los activos intangibles que tengan una vida útil indefinida no sean amortizados, aunque tendrán que ser revisados anualmente. En esta misma norma también se autoriza a que los activos intangibles que tengan una vida útil finita se amorticen durante la misma, pero sin la restricción de un límite máximo.

\footnotetext{
${ }^{1}$ En la actualidad, el International Accounting Standard Board (IASB) publica sus normas a través de una serie de pronunciamientos denominados IFRS (International Financial Reporting Standards). Este organismo ha adoptado el cuerpo de normas emitidas por el IASC, cuyos pronunciamientos continúan designándose como IAS (International Accounting Standards).
} 
Similarmente, en el ámbito internacional el IASC recomienda que las empresas capitalicen los costes de los intangibles siempre y cuando se cumplan los tres siguientes requisitos (NIC 38 del IASC [1998]): el coste puede ser medido con fiabilidad, el activo intangible es controlable por la empresa y se puede identificar con independencia del fondo de comercio, el activo está asociado con probables beneficios futuros.

Así, los intangibles reconocidos, ya sean adquiridos externa o internamente, deberán amortizarse durante la mejor estimación de su vida útil, siendo la recomendación de que no supere los 20 años. Por otro lado, debemos indicar que la revalorización de un intangible solo será posible si existe un mercado activo para el mismo. Otra de las principales novedades introducidas por el IASC es el referente a la recomendación de divulgar información detallada sobre los mismos. No obstante, si atendemos a los requisitos exigidos, muchos de los gastos de $\mathrm{I}+\mathrm{D}$, gastos de establecimiento y gastos de publicidad deberían ser cargados a resultados en el periodo en el que se producen.

En cuanto al Reino Unido, y de forma similar a la normativa del FASB, el Accounting Standards Board (ASB) indica que los costes de los activos intangibles adquiridos externamente, incluido el fondo de comercio, deben registrarse como activos y amortizarse durante su vida útil. Se presume que la vida útil de estos intangibles se limita a los 20 años, y que el coste de los intangibles internamente desarrollados, como los de $\mathrm{I}+\mathrm{D}$, deben llevarse a resultados en el momento en que se incurre en ellos.

El caso español es similar a lo hasta aquí expuesto, de manera que existe una normativa estricta en el reconocimiento y amortización de los intangibles, siendo el límite máximo de amortización del fondo de comercio de veinte años. Sin embargo, un caso peculiar es el de Australia, en el que la contabilización de los intangibles siempre ha estado desregulada, dado que no existen normas para la mayoría de intangibles. Según Goodwin [2002], en este país las empresas tienen relativa libertad para capitalizar o llevar a resultados el coste de los intangibles. A su vez, es posible la revalorización de los activos intangibles, así como el reconocimiento de aquellos internamente desarrollados en la empresa. Ambos aspectos no están permitidos bajo los principios contables de Estados Unidos, salvo los mencionados costes de desarrollo de software según la SFAS nº 86.

En definitiva, en la gran parte de países se imponen grandes restricciones a la aparición de activos intangibles en los balances de las empresas. Esto se debe, principalmente, a la dificultad en la identificación de muchos de ellos, a la dificultad en su valoración, y a la incertidumbre en las expectativas de generación de beneficios de 
los mismos. Si su reconocimiento en el activo aporta o no utilidad para los inversores a la hora de fijar el valor de la empresa es una cuestión que se ha estudiado empíricamente a lo largo del tiempo. A continuación nos referimos a estos estudios ${ }^{2}$.

\subsection{Revisión de la literatura}

La continua controversia que rodea la contabilización de los intangibles ha centrado la atención de los investigadores académicos en el ámbito internacional. La mayoría de estas investigaciones han tratado aspectos relacionados con el fondo de comercio proveniente de las adquisiciones de empresas, que es el mayor intangible reconocido en la mayoría de empresas. Estudios como los de Amir, Harris y Venutti [1993], Chauvin y Hirschey [1994] y McCarthy y Schneider [1995] documentan la existencia de una relación positiva y significativa entre el fondo de comercio y el valor de mercado de la empresa.

Otros trabajos que han utilizado medidas concretas de intangibles son los de Ittner y Larcker [1998], Barth, Clement, Foster y Kasznik [1998] y Black, Carnes y Vernon [1999], que confirman que éstos están relacionados con los precios de mercado. Para ello, utilizan medidas de la satisfacción del consumidor, valoración de marcas y reputación de la empresa, respectivamente. Por su parte, Lev y Sougiannis [1996] muestran que los precios de las acciones reflejan los activos intangibles, al menos parcialmente, al encontrar una relación positiva entre los precios y estimaciones de activos de $\mathrm{I}+\mathrm{D}$ basadas en los gastos de $\mathrm{I}+\mathrm{D}$ de cada una de las empresas de su muestra. A su vez, Amir y Lev [1996] encuentran evidencia de que el patrimonio contable y el resultado de las empresas con niveles significativos de activos intangibles tienden a estar infravalorados con relación a sus valores de mercado.

También los resultados del estudio de Jennings, Robinson, Thompson y Duvall [1996] confirman la evidencia anterior, de manera que los inversores valoran el fondo de comercio como un activo más de la empresa. Sin embargo, este último estudio encuentra escasa evidencia de la existencia de una relación sistemática entre la amortización del fondo de comercio y los valores de mercado. Para Choi, Kwon y Lobo [2000] esta escasa relación se debe a que dicha amortización, según los principios

\footnotetext{
${ }^{2}$ Una completa revisión de la literatura sobre intangibles puede encontrarse en Cañibano, García-Ayuso y Sánchez [1999]
} 
contables generalmente aceptados, no refleja adecuadamente la pérdida de valor del activo intangible durante el periodo.

Por ello, Choi, Kwon y Lobo [2000] proponen una metodología de formación de carteras para examinar la valoración por parte del mercado estadounidense tanto de los activos intangibles reconocidos como del gasto asociado por amortización de los mismos. Concretamente el estudio realizado por estos autores se basa en la comparación de los ratios book-to-market $(\mathrm{B} / \mathrm{M})$ y earnings-price $(\mathrm{E} / \mathrm{P})$ para una cartera de empresas con activos intangibles significativos y estables y una cartera de control de empresas sin activos intangibles. A su vez, para complementar este análisis y determinar la relevancia de los intangibles y su gasto asociado por amortización, realizan un análisis de regresión en corte transversal similar al de Jennings, Robinson, Thompson y Dubai [1996].

Durante el periodo 1978-1994 los resultados del estudio de Choi, Kwon y Lobo [2000] confirman que los mercados financieros valoran positivamente los activos intangibles divulgados por las empresas en sus estados financieros. No obstante, obtienen evidencia de que la valoración por parte de los inversores de un dólar de activo intangible es significativamente inferior a la valoración del resto de activos contabilizados, lo que es consistente con la hipótesis de incertidumbre en las expectativas de generación de beneficios futuros. A su vez, el análisis de la valoración por parte del mercado del gasto por amortización de intangibles, indica que aunque el mercado no valora el gasto por amortización de forma significativa, sí que tiene en cuenta de forma distinta el gasto por amortización en relación con otros gastos de la cuenta de resultados. En definitiva, para estos autores el estudio empírico apoya las peticiones de que los activos intangibles sean reconocidos en los balances de las empresas, pero no se requiere que sean amortizados para reflejar su supuesta pérdida de valor. Esto último es consistente con la visión de los inversores de que los activos intangibles no sufren depreciación sistemática o bien de que el gasto por amortización refleja la pérdida de valor de los intangibles con un error considerable.

Por tanto, la evidencia de todos estos estudios, desarrollados en el entorno estadounidense, es clara en cuanto a la existencia de una relación positiva entre activos intangibles y precio de mercado. A su vez, creemos también interesante revisar tres casos concretos en el entorno de Estados Unidos: el de las empresas de desarrollo de software, el del sector bancario, y el de las empresas de la década de 1920, antes del establecimiento en Estados Unidos de la Securities and Exchange Commission (SEC).

Es el trabajo de Abbody y Lev [1998] el que trata de estudiar la norma estadounidense sobre capitalización de los costes de desarrollo de software (SFAS no 
86). Esta norma proporciona la posibilidad de estudiar el tratamiento contable de este tipo de intangible, que difiere de la imputación completa a resultados del resto de intangibles.

A su vez, estos autores tratan de explicar dos posiciones manifestadas por colectivos implicados en esta norma. Por un lado, la Software Publishers Association (SPA) solicitó en 1996 la abolición de la citada norma, puesto que, para esta asociación, y dados los cambios producidos en la industria desde que se aprobó la norma en 1986, la capitalización de los costes de desarrollo de software no beneficia a los inversores. Su razonamiento lo basan en la existencia de gran incertidumbre en la viabilidad tecnológica y económica del software en proceso de desarrollo, de manera que los usuarios de la información financiera no creen que este activo sea relevante. Es más, para la SPA los usuarios de la información son muy escépticos a esta capitalización y prefieren su cargo inmediato a resultados del ejercicio, concluyendo que "los estados financieros serían más fiables y consistentes si todos los costes de desarrollo de software se cargaran a resultados" (véase Aboody y Lev [1998, p. 162]). Esta petición de abolición de la norma es sorprendente, si tenemos en cuenta dos aspectos. Primero, que fue la propia asociación de empresas de software existente en 1985 (The Association of Data Processing Service Organizations) la que apoyó la emisión de la norma. A su vez, esta norma es lo suficientemente flexible como para que los directivos puedan elegir entre capitalizar o llevar a resultados los costes de desarrollo de software, por lo que no necesitarían su abolición para cargar a resultados estos costes. Por otro lado, los analistas financieros también han mostrado la objeción a la capitalización del software. Esta posición también es sorprendente, pues dicha capitalización puede ser fácilmente deshecha restando el importe capitalizado del resultado de la empresa y del activo total. Es decir, para Aboody y Lev [1998] la capitalización siempre tiene contenido informativo sobre el éxito de la empresa, puesto que en el peor de los casos esta información puede ser ignorada.

Por todo ello, estos autores, mediante una muestra de 163 empresas en el periodo 1987-1995, examinan la relevancia de la información sobre la capitalización de este intangible mediante tres procedimientos: el análisis de la asociación de las rentabilidades de mercado con la información financiera, el análisis de la asociación de los precios de mercado con dicha información, y el análisis de la capacidad predictiva de dicha información sobre los resultados futuros.

Los resultados de su estudio muestran que los costes de desarrollo de software capitalizados están relacionados positivamente con las rentabilidades de mercado. De 
igual forma, el activo intangible que se va formando a través de dichas capitalizaciones también está asociado con los precios de mercado. Y lo que es más importante, dicha capitalización está asociada con los beneficios futuros, no encontrándose evidencia de que la subjetividad que implica la capitalización o no de estos costes haga decrecer la calidad del beneficio. Por último, estos autores documentan la existencia de una relación significativa entre los costes de desarrollo de software directamente cargados a resultados por las empresas que deciden no capitalizarlos y las rentabilidades futuras de mercado, lo que es consistente con una reacción retardada de los inversores hacia el desarrollo de productos de estas empresas. La explicación que encuentran los autores a este retardo se basa en que ante las empresas que no capitalizan el software, los inversores no pueden inicialmente distinguir entre proyectos que superan la fase de viabilidad y proyectos que han fracasado. Por ello, la resolución final de esta incertidumbre lleva a la obtención de rentabilidades de mercado positivas. Esta reacción retardada no se da en las empresas que capitalizan, ya que en ellas la información sobre la capitalización afecta al precio de forma contemporánea. Así pues, los resultados del estudio no apoyan las afirmaciones de la SPA sobre la irrelevancia de la capitalización del software.

En cuanto a la objeción de los analistas a la capitalización del software, ésta debe estar relacionada con el elemento aleatorio que introduce la misma, ya que la capitalización de una cantidad desconocida de un componente tan importante en estas empresas dificulta en gran medida la tarea de predecir. Otra posibilidad es que los analistas con buenas relaciones con algunas empresas pueden obtener información privada sobre el éxito o fracaso de los proyectos, de forma que la divulgación parcial de esta información les impida aprovechar esta ventaja. Así, los autores introducen en el análisis datos sobre las predicciones de los analistas para investigar la motivación de sus afirmaciones en contra de la capitalización. Controlando por el tamaño de las empresas, el horizonte de predicción y el número de analistas que las siguen, Abbody y Lev [1998] encuentran evidencia de que los errores de predicción de los analistas están positivamente relacionados con la intensidad en la capitalización del software. Por ello, la evidencia es consistente con la posibilidad de que la objeción de los analistas a la capitalización se deba al efecto adverso que tiene la misma sobre la calidad de sus predicciones de beneficios.

Por su parte, Kohlbeck y Warfield [2002] centran su estudio en el sector bancario, tratando de analizar el impacto de los activos intangibles no reconocidos sobre el valor de las acciones de la empresa, dentro del contexto de la existencia de resultados anormales del modelo de Ohlson [1995]. Según estos autores, el estudio de este sector 
es importante, pues la información revelada por los bancos en Estados Unidos permite derivar estimaciones razonables de activos intangibles concretos. Para la medición de estos activos intangibles no reconocidos, estos autores utilizan técnicas de descuento de flujos basadas en metodologías utilizadas por expertos en la valoración de intangibles relacionados con los servicios de préstamos, las tarjetas de créditos, los depósitos y los fondos de inversión.

Mediante un análisis de regresión, los resultados indican la existencia de una asociación positiva y significativa entre el nivel de intangibles no reconocidos y los resultados anormales. Además, encuentran evidencia de la existencia de diferencias significativas en la forma de valorar los bancos, según los niveles de intangibles no reconocidos. Concretamente, la importancia de los resultados anormales en el valor de la empresa se incrementa en las empresas con mayores niveles de intangibles no reconocidos. En definitiva, el estudio subraya la importancia de los activos intangibles y muestra la influencia en los precios de mercado de aquellos no reconocidos en balance.

En cuanto al último de los casos, el de las empresas cotizadas en Estados Unidos con anterioridad a la aparición de la SEC, debemos comentar el trabajo de Ely y Waymire [1999], que analiza la relación entre precios de mercado y activos intangibles contabilizados en un entorno donde los directivos de las empresas disponían de cierta discrecionalidad para capitalizar tales activos. El estudio utiliza datos de 1927, cuando muchas empresas mostraban gran cantidad de activos intangibles en sus balances, debido a que los directivos podían capitalizar un amplio abanico de intangibles, así como decidir su política de amortización y divulgación de la información.

Entre los resultados más relevantes, debemos destacar que no se encuentra evidencia de la existencia de una relación positiva entre intangibles capitalizados y precio de las acciones. Por el contrario, sí que hay evidencia de que la relevancia de los resultados contables es una función decreciente del nivel de intangibles capitalizados. Este resultado estaría señalando la percepción por parte de los inversores de que las empresas obtienen mayores resultados a través de la capitalización de intangibles que deberían haber sido contabilizados como gasto del ejercicio. Así, los autores concluyen que los inversores tenían una visión escéptica de los activos intangibles, por ello, "la evidencia sugiere que proporcionar a los directivos de mayor libertad para capitalizar intangibles puede que no incremente de forma clara la cantidad de información fiable disponible para los inversores" (Ely y Waymire [1999, p. 19]).

A continuación vamos a destacar un par de trabajos realizados en un contexto menos regulado que el de Estados Unidos, como es el de las empresas australianas. En 
primer lugar, Barth y Clinch [1998] tratan de determinar si las revalorizaciones de los activos financieros, tangibles e intangibles hacia su valor razonable, son tenidas en cuenta por los inversores. Los resultados indican una relación positiva entre los intangibles capitalizados (y sus posteriores revalorizaciones) y los precios de mercado.

Por otro lado, Goodwin [2002] lleva a cabo un interesante estudio en el entorno contable de Australia, con objeto de determinar la relevancia valorativa de los intangibles, y para confirmar si el supuesto declive en la relevancia de los resultados en Estados Unidos puede deberse a las limitaciones sobre la capitalización de los intangibles en este último país. Este autor realiza un estudio en corte transversal con datos del periodo 1975-1999, obteniendo alguna evidencia de la disminución de la relevancia valorativa del resultado contable, lo que es consistente con los estudios estadounidenses. Sin embargo, sólo las pérdidas en la cuenta de resultados parecen explicar la merma en la relevancia. Además, análisis posteriores en los que el autor diferencia entre empresas que reconocen y no reconocen intangibles, muestran sólo el declive de la relevancia de la información suministrada por estas últimas. En definitiva, para este autor los resultados confirman las afirmaciones de Lev y Zarowin [1999] en el sentido de que los resultados de las empresas que capitalizan intangibles reflejan relativamente más información para la valoración de la empresa que las empresas que no los capitalizan. A su vez, las empresas que amortizan los intangibles obtienen una relevancia mayor que las que no amortizan, estando esta amortización y su variación anual significativamente asociada con la rentabilidad de mercado de la empresa, lo que sería indicativo de su contenido informativo sobre los resultados futuros.

En cuanto a los estudios realizados en nuestro país, debemos destacar el de Larrán, Monterrey y Mulero [2000], que estudian la relevancia valorativa del fondo de comercio en el periodo 1991-1997. Los resultados muestran que el fondo de comercio es percibido en el mercado como un activo más, asociado a la creación de valor de la empresa. A su vez, se obtiene evidencia de un incremento en el tiempo de la relevancia valorativa del fondo de comercio en el mercado bursátil, así como la dependencia de dicha relevancia de factores contextuales como el nivel de resultados y la magnitud relativa del fondo de comercio. Igualmente, Cañibano, García-Ayuso y Sánchez [2000] tratan el problema de la medición de la innovación empresarial en el contexto español y la valoración de los intangibles en los estados financieros. Su estudio empírico en el periodo 1990-1996 encuentra evidencia de que, por término medio, el mercado español valora a las empresas por encima de sus valores contables, siendo el ratio $\mathrm{B} / \mathrm{M}$ menor cuanto mayor es el nivel tecnológico de la empresa. 


\section{Metodología}

En este apartado vamos a exponer la metodología que se va a emplear en el estudio empírico para alcanzar los objetivos propuestos. En este sentido, vamos a diferenciar dos procedimientos distintos. En una primera fase, tratamos de establecer la relevancia valorativa de los activos intangibles a través de un análisis de regresión. Basándonos en este resultado propondremos una medida aproximativa de los activos intangible no reconocidos. Con esta información, y con una metodología de carteras, analizaremos las rentabilidades obtenidas siguiendo distintas estrategias de inversión.

\subsection{Relevancia valorativa de los activos intangibles reconocidos}

El trabajo de Ohlson [1995] ha estimulado un cuerpo creciente de trabajos de relevancia que examina el vínculo entre el valor de mercado de las empresas y las cantidades reconocidas en los estados financieros. Las conclusiones del mismo indican que el valor contable de los fondos propios y el resultado contable son las dos variables fundamentales para los usuarios de la información financiera, y que deben determinar y conducir los precios de mercado de las acciones de las empresas. A su vez, también se incluye la "otra información", que es información conocida sobre los resultados futuros pero que aún no está reflejada en el balance y cuenta de resultados.

Así, si nos centramos en los últimos años, podemos observar que muchos estudios tratan de determinar la relevancia de las variables contables utilizando regresiones del precio de mercado sobre variables como el resultado neto y el valor contable de los fondos propios, considerando la siguiente regresión de niveles:

$$
P_{i t}=\alpha_{0}+\alpha_{1} B V_{i t}+\alpha_{2} X_{i t}+\varepsilon_{i t}
$$

donde, para cada empresa i:

$\mathrm{P}_{\mathrm{t}}$ : valor de mercado de las acciones en el momento $\mathrm{t}$

$\mathrm{BV}_{\mathrm{t}}$ : valor contable de los fondos propios o patrimonio contable en el momento $t$

$X_{t}$ : resultado contable del periodo $(t-1, t)$

Como ejemplos de aplicación en la literatura, podemos destacar, entre otros muchos, los trabajos de Amir y Lev [1996], que estudian la relevancia valorativa de la 
información contable y de otros indicadores no financieros en las empresas de telefonía móvil; Aboody [1996] que tratar de investigar la valoración por parte del mercado de las opciones sobre acciones de los empleados de una empresa; Burgsthaler y Dichev [1997] y Barth, Beaver y Landsman [1998], que se centran en la importancia del patrimonio contable y del resultado según la situación financiera de la empresa, haciendo especial mención al valor de la empresa bajo la opción de liquidación o abandono; Collins, Maydew y Weiss [1997] y Francis y Schipper [1999] que examinan la evolución de la relevancia valorativa del patrimonio contable y del resultado a lo largo de cuatro décadas; y Lev y Zarowin [1999] que documentan el declive en el tiempo de la relevancia del patrimonio contable en contraste con el incremento en la importancia de los activos intangibles.

De este modo, la relación entre el precio de las acciones y los activos intangibles contablemente reconocidos durante el periodo 1991-1999 se realiza a través de las siguientes regresiones en corte transversal:

$$
\begin{aligned}
& P_{i t}=\gamma_{0}+\gamma_{1} B V_{i t}+\gamma_{2} X_{i t}+\varepsilon_{i t} \\
& P_{i t}=\gamma_{0}+\gamma_{1} \operatorname{Tg} B V_{i t}+\gamma_{2} X_{i t}+\gamma_{3} \operatorname{Intg}_{i t}+\varepsilon_{i t}
\end{aligned}
$$

donde, además de las variables antes definidas, para cada empresa i:

$\mathrm{TgBV}_{\mathrm{it}}$ : valor contable de los activos tangibles en el periodo $\mathrm{t}$

Intg $_{\text {it }}$ : Activos intangibles reconocidos en el balance en el periodo $t$

En la primera regresión tratamos la relevancia valorativa de las dos variables contables relevantes en el contexto anteriormente citado de Ohlson [1995]: el resultado contable y el valor contable de los fondos propios. Los resultados esperados deben indicar la significatividad de ambas variables, presentado ambas signos positivos, lo que confirmaría la hipótesis de relevancia de dichas variables.

Sin embargo, en la segunda regresión, el valor contable total (BV) se descompone en dos componentes: el valor contable de los elementos tangibles (TgBV), y el valor contabilizado de activos intangibles (Intg). Tratamos de contrastar dos hipótesis. Por un lado, la relevancia valorativa del resultado contable y el valor contable de los elementos tangibles. Coeficientes positivos y significativos para estas variables confirmarían este hecho. A su vez, el coeficiente sobre los activos intangibles reconocidos debe presentar un valor positivo y significativo, siempre y cuando los 
inversores perciban dichos intangibles como activos legítimos de la empresa y no como activos inexistentes provenientes de la manipulación de la empresa de su cuenta de resultados al capitalizar gastos. De esta forma, los activos intangibles reconocidos proporcionarían información sobre los futuros beneficios de la empresa, contribuyendo a los mismos. La confirmación de esta hipótesis corroboraría que a pesar de los problemas para su medición, identificación y reconocimiento, los inversores ponderan estos activos de forma positiva.

A su vez, creemos conveniente incluir una variable adicional que muestre la interacción entre los beneficios de la empresa y sus activos intangibles reconocidos.

$$
P_{i t}=\gamma_{0}+\gamma_{1} \operatorname{TgB} V_{i t}+\gamma_{2} X_{i t}+\gamma_{3} \operatorname{Intg}_{i t}+\gamma_{4}\left(X_{i t} \cdot \operatorname{Intg}_{i t}\right)+\varepsilon_{i t}
$$

Siguiendo a Ely y Waymire [1999], si los inversores perciben los activos intangibles como un mero indicador de gastos diferidos que deberían de haber sido directamente cargados a resultados, entonces la interacción resultado-intangible presentará una relación negativa con los precios observados en el mercado. Esto estaría indicando que el coeficiente de respuesta al resultado disminuye con el nivel de activos intangibles, es decir, el efecto debería quedar más patente cuanto mayor es la magnitud del importe capitalizado. Así, bajo esta premisa los inversores no estarían dando tanta relevancia al resultado obtenido por la empresa, ya que estaría sobrevalorado por la capitalización de intangibles. El signo y significatividad del coeficiente $\gamma_{4}$ permitirá contrastar esta hipótesis.

Por último, debemos mencionar el llamado efecto escala, que es uno de los problemas econométricos identificados en los estudios en sección cruzada en los que la variable dependiente son los precios de mercado y las variables independientes son los datos contables. Este efecto se produce por la existencia de diferencias de escala o tamaño entre las distintas unidades económicas, de manera que se observa que las empresas grandes (pequeñas) presentan valores grandes (pequeños) en la mayoría de las variables contables y financieras. Por ello, los resultados de las regresiones podrían estar condicionados por la submuestra de empresas más grandes, que, sin embargo, está normalmente integrada por menos casos.

La literatura previa que ha tratado el efecto escala indica que se pueden producir sesgos en las estimaciones de los coeficientes y en los errores estándar. A su vez, Brown, Lo y Lys [1999] muestran que el $\mathrm{R}^{2}$ del modelo estimado también está sesgado cuando se produce el efecto escala. En este sentido, cuando el efecto escala es lo 
suficientemente grande, en realidad se está regresando la escala sobre ella misma, por lo que el $\mathrm{R}^{2}$ se aproxima a uno.

Desgraciadamente, identificar la escala es una tarea difícil, por lo que a menudo se aproxima por alguna variable supuestamente correlacionada con ella, siendo varias las alternativas apuntadas por la literatura. Así, Barth y Kallapur [1996, p. 528] apuntan que para reducir los sesgos en las estimaciones se podrían deflactar las variables por un subrogado de la escala o incluir dicho subrogado como variable independiente que controle los efectos de la misma. Por otra parte, para corregir la heterocedasticidad de los errores de la regresión se pueden utilizar los errores estándar consistentes ante heterocedasticidad de White [1980].

No existe un consenso de las investigaciones contables sobre qué variable utilizar como subrogado, siendo las comúnmente consideradas en la literatura contable; el activo total, el valor contable de los recursos propios, el resultado, el número de acciones y el precio de mercado. Brown, Lo y Lys [1999] y Easton y Sommers [2000] muestran que utilizar datos por acción no resuelve el efecto escala, puesto que el número de acciones y su valor nominal es un número totalmente arbitrario elegido por la dirección de la empresa, lo que lleva al problema de la existencia de una escala inconsistente, esto es, se mezclan distintas unidades de medida dentro de un mismo modelo, ya que algunas acciones son muy grandes y otras muy pequeñas. Además, las políticas de splits o desdoblamiento de acciones provoca que en una misma empresa la escala varíe en el tiempo. En cambio, para estos autores, si se utiliza el precio de mercado al principio del periodo, sí que pueden resolverse los problemas de escala, ya que el precio es una variable que refleja el tamaño de una acción.

Por todo ello, en el presente estudio hemos seguido las recomendaciones mencionadas, utilizando el precio de mercado al principio del periodo como deflactor en las regresiones [2] a [4], y utilizando los errores estándar consistentes ante heterocedasticidad de White [1980].

\subsection{Medición de los activos intangibles no reconocidos}

Una vez estimado el modelo [3] anterior, proponemos medir la cantidad de activos intangibles no reconocidos a través del residuo de esta regresión. Es decir, estos intangibles no reconocidos serían la diferencia entre el valor de mercado de la empresa y el valor dado por los inversores según los coeficientes estimados, las variables contables básicas y los activos intangibles que sí están recogidos en el balance. 
No obstante, debemos indicar que si medimos los intangibles no reconocidos como el residuo de la regresión, no estamos midiendo exactamente esta variable, ya que la estimación de la regresión minimiza la suma de residuos al cuadrado, por lo que habrá tanto errores positivos como negativos que ajustarán los valores de la muestra a la recta de la regresión. Por ejemplo, consideremos el caso ficticio de una empresa con un valor nulo de recursos propios, beneficio, activos intangibles y valor de mercado. Evidentemente, sus activos intangibles no reconocidos serían cero. Sin embargo, si tomamos el residuo de la regresión, el valor de los intangibles no reconocidos de esta empresa sería exactamente igual al intercepto de la regresión, esto es, $-\gamma_{0}$. Es decir, el intercepto de una regresión recoge el valor medio de las variables omitidas en el modelo, en este caso los activos intangibles no reconocidos en la contabilidad y que sí son valorados por el mercado de capitales. Si incluimos este intercepto en el cálculo de los intangibles no reconocidos estamos restando este valor medio de todas las empresas, valor que por otra parte no aparece de ningún modo en la información económicafinanciera de las empresas.

Por ello, si queremos obtener una medida completa de estos activos intangibles no reconocidos debemos sumar este valor medio a los residuos, por lo que la expresión finalmente utilizada es la siguiente ${ }^{3}$ :

$$
\operatorname{IntgNo} \operatorname{Re} c=P_{i t}-\left(\hat{\gamma}_{1} \operatorname{TgB} V_{i t}+\hat{\gamma}_{2} X_{i t}+\hat{\gamma}_{3} \operatorname{Intg}_{i t}\right)
$$

De esta forma, al no incluir el intercepto, podremos comparar el valor de mercado de la empresa con el valor resultante de sus datos contables fundamentales (valor contable de los recursos propios correspondiente a los elementos tangibles, resultado contable e intangibles reconocidos). La existencia de una empresa con un alto valor de mercado en relación con sus variables contables nos estaría indicando la presencia de otros elementos de valoración con potencial de obtención de beneficios futuros que no han sido incluidos en su balance o cuenta de resultados. Además, una empresa que no active los intangibles, llevándolos sistemáticamente a resultados, obtendrá un mayor valor de intangibles no reconocidos, ya que no aparecerá como activo y el resultado contable será menor. En definitiva, esta medida debería estar

\footnotetext{
${ }^{3}$ No obstante, el incluir o no el intercepto no influye en ningún caso al estudio empírico, pues no tiene ninguna influencia en la formación de carteras sumar o restar un valor constante a todas las observaciones de la muestra.
} 
altamente correlacionada con los activos intangibles no reconocidos, variable que no es observable directamente en la información financiera facilitada por las empresas.

\subsection{Análisis de rentabilidades}

Como se ha comentado anteriormente, uno de los objetivos de la investigación es analizar si el nivel de activos intangibles, tanto aquellos reconocidos como los no reconocidos contablemente, supone una mayor incertidumbre para el inversor. Esto llevaría a la exigencia por parte de los mismos de una mayor rentabilidad de mercado.

Para comprobar esta hipótesis realizamos un análisis de rentabilidades a partir de la formación de carteras basadas en determinadas pautas de inversión según los niveles de intangibles. Tendremos en cuenta el trabajo de Aboody y Lev [1998], que documenta la existencia de mayores errores en las predicciones de beneficios de los analistas para las empresas de Estados Unidos que capitalizan los costes de desarrollo de software. Así, podemos extender este análisis a nuestro país y a todas los sectores en general, comprobando si la magnitud de estos errores influyen en las rentabilidades obtenidas. Equivalentemente, y al igual que Aboody y Lev [1995], tendremos en cuenta el número de analistas que siguen a las empresas como variable de control, puesto que existe evidencia de que la exactitud de las predicciones de los analistas es mayor para las empresas más seguidas por los mismos (véase Lys y Soo [1995]).

Así, en primer lugar, tomaremos como referencia el nivel de activos intangibles que sí se hallan reconocidos en los balances de las empresas, formando diez carteras en función de esta variable en cada uno de los años del periodo 1992-1999, y calculando la rentabilidad media pura y ajustada por riesgo de cada una de las carteras. La existencia de diferencias en las rentabilidades obtenidas sería indicativa de la diferente percepción de los inversores de las empresas con mayores y menores intangibles contabilizados. A continuación, se indagará las posibles interacciones existentes entre ambas características, activos intangibles reconocidos y errores de predicción, puesto que para un determinado nivel de intangibles, se podría observar que la magnitud de los errores de predicción influyen de manera diferente que para otro.

Una vez realizado este análisis para los activos intangibles reconocidos, en segundo lugar, seguiremos este mismo procedimiento, pero basándonos en el nivel de activos intangibles no reconocidos. De esta forma, se podrá estudiar si el comportamiento del mercado ante la existencia de atributos de valor intangibles que no están reconocidos contablemente difiere de los que sí se hallan contabilizados. Por 
último, volveremos a aplicar el mismo procedimiento, pero utilizando el ratio $\mathrm{B} / \mathrm{M}$ como subrogado de los elementos intangibles no reconocidos, puesto que este ratio también debe ser indicativo de la existencia de elementos intangibles relevantes no contabilizados. Las conclusiones de los trabajos de Choi, kwon y Lobo [2000] y Cañibano, García-Ayuso y Sánchez [2000] así lo indican.

\section{Selección y análisis de la muestra}

\subsection{Variables utilizadas en el estudio empírico}

Para la realización del estudio empírico se necesita disponer de datos contables, datos procedentes de los analistas financieros e información de las cotizaciones bursátiles. Para ello, hemos utilizado hasta tres fuentes de información distintas.

\section{Variables procedentes de los estados financieros}

Para la obtención de la información contable se dispone de la información pública periódica que las empresas cotizadas deben depositar en la $\mathrm{CNMV}^{4}$. El periodo disponible abarca los cierres de los ejercicios 1991 a 1999. En este sentido, para poder llevar a cabo el estudio necesitamos cuatro de estas variables: por un lado, las dos variables fundamentales para la valoración, es decir, el valor contable de los recursos propios y el resultado del ejercicio; y por otro lado, el activo total y los activos intangibles reconocidos en el balance.

Antes de indicar las partidas concretas que se han tomado para cada periodo, debemos resaltar que se ha considerado la información contable consolidada para las empresas que han presentado este tipo de información. En el caso de las empresas que sólo han presentado información individual, ésta es la que se ha utilizado en el estudio empírico. Hecha esta matización, como valor contable de los recursos propios tomamos para cada cierre de ejercicio del periodo 1991-1999 el epígrafe "Fondos Propios" de la base de datos de la CNMV, que está compuesto por las siguientes partidas:

\footnotetext{
${ }^{4}$ Disponible en su página web: http://www.cnmv.es
} 
+ capital suscrito

+ reservas de la sociedad dominante

+ reservas sociedades consolidadas

$+/$ - diferencias de conversión

+/- resultados atribuibles a la sociedad dominante

- Dividendos a cuenta entregados en el ejercicio

$=$ Fondos propios

En cuanto al resultado del ejercicio, hemos tomado el resultado neto después de impuestos, que en el formato de la base de datos de la CNMV viene representado en la cuenta de resultados bajo la partida de "Resultado de la Sociedad Dominante". Debemos indicar que este resultado después de impuestos es menos sensible a la posible manipulación clasificatoria de las partidas de la cuenta de resultados.

Por último, se han sumado las partidas "Gastos de Establecimiento", "Inmovilizado Inmaterial" y "Fondo de Comercio Consolidado" para obtener los activos intangibles netos reconocidos. Estos activos intangibles reconocidos han sido divididos por el activo total para hallar la intensidad de las inversiones en intangibles reconocidos. El valor contable de los elementos tangibles se ha calculado como la diferencia entre los fondos propios y los activos intangibles netos reconocidos.

\section{Variables procedentes de los mercados financieros}

Para la ejecución de los distintos contrastes, así como para la obtención de las distintas rentabilidades de las carteras que se formarán en el estudio empírico, ya sean puras o ajustadas por riesgo, necesitamos la siguiente información. Por un lado, para el cálculo de las rentabilidades mensuales y anuales se han necesitado las series de cotizaciones de precios, de derechos de suscripción y de pago de dividendos, así como información sobre el desdoblamiento de acciones. Esta información se ha tomado de la base de datos disponible en nuestro Departamento, que se ha generado a partir de datos de las acciones que cotizan en el mercado continuo y que se han ido recopilando de distintas fuentes. Las rentabilidades mensuales se han calculado a partir de los precios de cierre del último día del mes, debidamente ajustadas por la existencia de derechos de suscripción, dividendos y desdoblamientos del valor nominal de las acciones dentro de ese mes. Las rentabilidades anuales se han calculado a través de la acumulación de las rentabilidades mensuales: $R_{\text {anual }}=\prod_{t=1}^{12}\left(1+R_{\text {mensual }, t}\right)-1$. 
Por otro lado, para el activo libre de riesgo se ha cogido para todo el periodo muestral el tipo de interés medio de los repos a un mes sobre Bonos del Estado, calculado a partir de la serie histórica del Boletín de la Central de Anotaciones publicada por el Banco de España en su página web ${ }^{5}$. A su vez, siguiendo las recomendaciones de Bartholdy y Peare [2001], se ha estimado la rentabilidad mensual de la cartera de mercado como la media simple equiponderada de las rentabilidades mensuales de todas las acciones del mercado continuo que cotizaron durante cada mes, hayan formado parte o no de la muestra final de este estudio.

\section{Variables procedentes de los analistas financieros}

La base de datos I/B/E/S (International Broker Estimate System) será el origen de la información acerca de las predicciones de beneficios por parte de los analistas. Esta base de datos contiene información completa y detallada de predicciones de resultados de las empresas españolas por parte de cada analista individual, así como datos generales sobre las empresas en el momento de realizar la predicción, como el precio de mercado, el número de acciones y el último beneficio revelado.

Para llevar a cabo el estudio utilizamos la siguiente información. Por un lado, para cada empresa y mes calculamos el número de analistas que siguen a las empresas del mercado continuo, es decir, el número de analistas que a lo largo del mes han realizado al menos una predicción de beneficios para el año en curso. Esta variable la hemos denominado "Atención". Si una empresa no fue seguida en algún mes se le ha dado un valor de cero.

Por otro lado, el producto del precio de la acción por el número de acciones nos da la capitalización bursátil de la empresa a finales de cada uno de los meses del periodo 1991-1999, variable que será utilizada en el estudio como subrogado del tamaño. El cociente entre el valor contable de los recursos propios (obtenido del balance de la empresa) y esta capitalización bursátil nos dará el valor del ratio Book-to-market $(\mathrm{B} / \mathrm{M})$.

A su vez, debemos indicar que $\mathrm{I} / \mathrm{B} / \mathrm{E} / \mathrm{S}$ ofrece el consenso de las predicciones de los analistas, es decir, la media y la mediana de todas las predicciones realizadas por cada analista para cada empresa y cierre fiscal. Este consenso es calculado por $\mathrm{I} / \mathrm{B} / \mathrm{E} / \mathrm{S}$ mensualmente, aproximadamente el tercer jueves de cada mes. De esta forma, hemos

\footnotetext{
5 http://www.bde.es
} 
calculado el error de predicción de los analistas a través de la siguiente expresión, que nos marca, en valor absoluto, la desviación relativa de las predicciones de los analistas:

Error de Predicción $=\mathrm{EP}=\left|\frac{x_{t+1}-f_{t}^{t+1}}{f_{t}^{t+1}}\right|$, donde:

$x_{t+1}$ : Resultado contable del periodo $(\mathrm{t}, \mathrm{t}+1)$

$f_{t}^{t+1}$ : Predicción del resultado contable del periodo $(\mathrm{t}, \mathrm{t}+1)$, realizado en el periodo $\mathrm{t}$

Debemos indicar que esta expresión se ha calculado anualmente en el periodo 1991-1999, tomando la primera predicción realizada por los analistas tras conocerse el resultado real del periodo anterior. Como consenso de la predicción del resultado contable por parte de los analistas se ha tomado la media de dichas predicciones para una misma empresa y cierre fiscal.

\subsection{Análisis descriptivo de la muestra}

El cruce de las tres bases de datos citadas en el apartado anterior nos deja una muestra final de 856 observaciones en el periodo 1991-1999, que se corresponden con 152 empresas distintas. A continuación describimos con detalle esta muestra con objeto de obtener una visión global de las variables utilizadas en el estudio empírico

La Tabla 1 muestra los valores medios y medianos de las variables utilizadas en el análisis de la relevancia valorativa de los activos intangibles. Dos resultados merecen destacarse. Por un lado, el valor contable de los fondos propios es claramente inferior al valor de mercado de la empresa, lo que es indicativo de ratios $\mathrm{B} / \mathrm{M}$ inferiores a la unidad. Por otro lado, las inversiones en activos intangibles reconocidas en el balance son muy pequeñas en términos relativos, pues menos de un $50 \%$ de la muestra presenta en sus balances unos intangibles superiores al 2\% del activo total. Este dato está muy alejado del 15-20\% obtenido por Ely y Waymire [1999] para las empresas estadounidenses en 1927, periodo en el que los directivos tenían menos limitaciones para la capitalización de intangibles.

En los últimos años la inversión en activos intangibles se ha convertido en una necesidad para el futuro éxito de las empresas. Si esto es así, y estos activos intangibles no han sido totalmente reconocidos en la contabilidad de las empresas españolas, la evolución del ratio $\mathrm{B} / \mathrm{M}$ debe mostrar una tendencia a la baja en el periodo analizado 
(véase Lev y Zarowin [1999]). Esto será así ya que cada vez una porción mayor y determinante del valor de la empresa no está reflejada en los estados financieros.

\section{Tabla 1. Estadística resumen de las variables contables utilizadas en el estudio}

La definición de las variables utilizadas puede observarse en el apartado cuarto. La intensidad en activos intangibles reconocidos se ha calculado como el cociente entre los activos intangibles reconocidos y el activo total. Los datos monetarios están expresados en miles de euros (tasa de conversión: 166,386 pesetas/euros).

\begin{tabular}{lrrr}
\hline t: Cierres fiscales años 1991-1999 & Media & Mediana & Observaciones \\
\hline Valor de mercado & $1.078 .827,55$ & $243.245,47$ & 856 \\
Valor contable de los fondos propios & $621.024,61$ & $162.639,89$ & 856 \\
Total activo & $1.773 .166,61$ & $397.262,99$ & 856 \\
Activos intangibles reconocidos & $90.221,53$ & $9.640,23$ & 856 \\
Intensidad en activos intangibles reconocidos & 0,0428 & 0,0189 & 856 \\
Resultado después de impuestos & $56.600,54$ & $14.586,56$ & 856 \\
\hline
\end{tabular}

Para comprobar esta hipótesis mostramos en la Tabla 2 la evolución en el tiempo del ratio $\mathrm{B} / \mathrm{M}$ y de los intangibles contabilizados. A su vez, trabajos previos, como el de Aboody y Lev [1998], han mostrado la reticencia de los analistas financieros a la capitalización de los gastos en activos intangibles debido a que hace más difícil su predicción al ser la incertidumbre una de las características fundamentales de los intangibles. Por ello, y al igual que estos autores, se incluye la evolución del error de predicción de los analistas, así como el número que por término medio han seguido a las empresas de la muestra, variables de control que serán utilizadas posteriormente.

Los resultados parecen corroborar las afirmaciones anteriores. Así, el ratio B/M ha experimentado un descenso progresivo a lo largo de la década de los 90, representado el valor contable menos del $60 \%$ del valor de mercado en los últimos 3 años analizados. Este descenso en el ratio $\mathrm{B} / \mathrm{M}$ se ha visto acompañado en los dos últimos años con el incremento en el porcentaje de intangibles reconocidos en los activos, aunque los mismos siguen representado un porcentaje modesto del total de activos. Sin embargo, en cuanto a los errores de predicción de las primeras estimaciones del ejercicio, se observa una mejora a partir de 1995 en la exactitud de los analistas. En definitiva, la falta de reconocimiento de activos intangibles en los últimos años puede ser la causa de que el ratio $\mathrm{B} / \mathrm{M}$ haya descendido en el tiempo, de manera que un porcentaje cada vez mayor del valor de mercado de las empresas no está representado en el balance de situación. Por ello, algunos investigadores han cuestionado recientemente la utilidad de la información financiera, sugiriendo la conveniencia de realizar cambios en la divulgación de información sobre intangibles. 
Tabla 2. Evolución en el tiempo del ratio $B / M$, la intensidad en activos intangibles reconocidos, el error de predicción y el grado de atención de los analistas

T: Año del cierre fiscal; B/M: Mediana del ratio book-to-market; Int Rec.: mediana del cociente entre los activos intangibles reconocidos y total activo; Error Pred.: mediana del valor absoluto del cociente (resultado real-predicción)/predicción; Atención: mediana del número de analistas que hacen predicciones de resultados para cada empresa de la muestra a lo largo del año t. El número de analistas que siguen a una empresa a lo largo del año se ha calculado como la media mensual de analistas que han realizado alguna predicción de beneficios en algún mes del año estudiado, esto es, la suma del número de analistas que realizan predicciones cada mes dividido por doce.

\begin{tabular}{crrrr}
\hline T & B/M & Int Rec. & Error Pred. & Atención \\
\hline 1991 & 1,0825 & 0,0133 & 0,1773 & 9,12 \\
1992 & 1,2684 & 0,0137 & 0,2763 & 10,04 \\
1993 & 0,8364 & 0,0157 & 0,2459 & 7,04 \\
1994 & 0,7310 & 0,0143 & 0,2140 & 8,33 \\
1995 & 0,8946 & 0,0163 & 0,1698 & 9,83 \\
1996 & 0,7607 & 0,0158 & 0,1429 & 10,92 \\
1997 & 0,5638 & 0,0162 & 0,1207 & 7,54 \\
1998 & 0,5116 & 0,0219 & 0,1308 & 7,75 \\
1999 & 0,5815 & 0,0351 & 0,1630 & 7,33 \\
\hline Total & $\mathbf{0 , 7 6 9 3}$ & $\mathbf{0 , 0 1 8 9}$ & $\mathbf{0 , 1 6 8 2}$ & $\mathbf{8 , 3 3}$ \\
\hline
\end{tabular}

Por último, podemos aportar mayor evidencia sobre este tema mediante la división de la muestra en cuatro carteras de igual número de datos en función de los activos intangibles reconocidos. Una vez realizadas estas carteras, podemos comprobar la existencia o no de diferencias significativas en los valores de las variables anteriormente comentadas. Los resultados, que se muestran en la Tabla 3, confirman que las empresas con menor porcentaje de activos intangibles reconocidos presentan un ratio $\mathrm{B} / \mathrm{M}$ superior a las empresas con mayor porcentaje de activos intangibles contabilizados, si bien debemos indicar que aunque la relación es estrictamente monótona y significativa para la mediana de las carteras, en el caso de la media esta relación solo se cumple para las dos carteras extremas. Al igual que en Choi, Kwon y Lobo [2000], parece que el mercado valora positivamente la existencia de activos intangibles reconocidos, ya que el ratio $\mathrm{B} / \mathrm{M}$ de la cartera de mayores intangibles reconocidos es significativamente inferior al de la cartera de menores intangibles.

Sin embargo, el nivel de activos intangibles no parece tener una relación clara con los errores de predicción de los analistas financieros. Así, no se observan diferencias significativas entre la media de las cuatro carteras. No obstante, este resultado aparece en consonancia con las afirmaciones realizadas por algunos analistas, pues se observa como en las empresas con menores activos intangibles reconocidos ha resultado más sencillo predecir sus beneficios. En las empresas con activos intangibles 
reconocidos (carteras 2 a 4) los errores de predicción son superiores, quizás debido a la mayor incertidumbre en cuanto a la capacidad de generar beneficios de estos activos. Resultados similares se obtienen con el grado de atención, pues el grupo de empresas con menores intangibles contabilizados es menos seguido por los analistas financieros en comparación con el resto de empresas.

Tabla 3. Ratio B/M, errores de predicción y grado de atención de los analistas según los cuartiles de activos intangibles reconocidos

Int Rec: Intangibles reconocidos, medidos como el cociente entre los activos intangibles reconocidos y activo total; B/M: Ratio book-to-market; Error Pred: Error de predicción, calculado como el valor absoluto del cociente (resultado real-predicción)/predicción; Atención: número de analistas que hacen predicciones de resultados para cada empresa de la muestra a lo largo del año t, calculado como la media mensual de analistas que han realizado alguna predicción de beneficios en algún mes del año estudiado, esto es, la suma del número de analistas que realizan predicciones cada mes dividido por 12 .

\begin{tabular}{|l|r|rr|rr|rr|}
\hline & \multicolumn{1}{|c|}{ Int Rec. } & \multicolumn{2}{|c|}{ B/M } & \multicolumn{2}{c|}{ Error Pred. } & \multicolumn{2}{c|}{ Atención } \\
\hline & Mediana & Mediana & Media & Mediana & Media & \multicolumn{1}{c|}{ Mediana } & Media \\
\hline Cartera 1 & 0,0018 & 0,8549 & 1,2639 & 0,1346 & 0,3726 & 7,71 & 10,33 \\
Cartera 2 & 0,0095 & 0,7861 & 0,8423 & 0,2731 & 0,6635 & 12,08 & 13,44 \\
Cartera 3 & 0,0281 & 0,7750 & 0,9521 & 0,1763 & 0,6597 & 12,17 & 13,39 \\
Cartera 4 & 0,0982 & 0,6424 & 0,7859 & 0,2037 & 0,5001 & 11,33 & 12,78 \\
\hline Contrastes: & & & & & & & \\
Diferencia entre & & & & & & & \\
carteras estadístico & $(3150,5)$ & $(11,25)$ & $(2,86)$ & $(10,16)$ & $(2,14)$ & $(8,57)$ & $(5,50)$ \\
p-valor & 0,000 & 0,010 & 0,036 & 0,017 & 0,093 & 0,036 & 0,001 \\
& & & & & & & \\
Diferencia carteras & & & & & & \\
1 - 4 estadístico & $(-18,59)$ & $(4,22)$ & $(3,23)$ & $(-2,31)$ & $(-1,29)$ & $(-3,36)$ & $(-2,72)$ \\
p-valor & 0,000 & 0,000 & 0,001 & 0,021 & 0,197 & 0,001 & 0,007 \\
\hline
\end{tabular}

Los contrastes de diferencias de medias y medianas entre las cuatro carteras están basados en el estadístico F de un test Anova y en el estadístico chi-cuadrado del Test de Pearson, respectivamente.

Los contrastes de diferencias de medias y medianas entre las carteras 1 y 4 están basados en el estadístico $\mathrm{F}$ de un Test Anova y el test de rangos de Wilcoxon, respectivamente. 


\section{Relevancia valorativa de los intangibles}

En este apartado pasamos a reflejar los resultados más importantes obtenidos en el estudio empírico, de acuerdo con la metodología expuesta en el apartado tercero.

\subsection{Activos intangibles reconocidos}

En primer lugar, analizamos los resultados de las estimaciones de los modelos [2] a [4], y que pueden observarse en la Tabla 4. La evidencia obtenida indica la existencia de una relación positiva y significativa entre el precio de mercado y las dos variables contables fundamentales: el valor contable de los fondos propios y el resultado del periodo. A su vez, la descomposición de dicho valor contable en los intangibles reconocidos en el activo y el valor contable del resto de elementos de la empresa confirma la relevancia de los activos intangibles, siendo su relación con el valor de mercado positiva y significativa. Por tanto, el primer resultado fundamental del análisis empírico, es consistente con la literatura previa y nos indica que los inversores tienen en cuenta esta variable a la hora de fijar el precio de las acciones.

En cuanto a la última de las regresiones, la presencia de un término de interacción no aporta evidencia de la posibilidad de que el coeficiente de respuesta del resultado contable sea una función decreciente del nivel de intangibles reconocidos. Así, aunque el coeficiente asociado a esta interacción es negativo, no es significativo, siendo su p-valor superior al $89 \%$. De esta forma, y dada las restricciones al reconocimiento de intangibles en los balances de las empresas españolas, los inversores no parecen considerar que un nivel alto de beneficios se deba a una capitalización en exceso de activos intangibles.

Además, creemos conveniente destacar un resultado, el coeficiente asociado a los activos intangibles es superior al coeficiente sobre el valor contable del resto de activos de la empresa. Esto supone que el mercado no considera que los futuros beneficios asociados a los intangibles contabilizados sean más inciertos que los futuros beneficios del resto de activos. Este resultado coincide con los obtenidos por Jennings, Robinson, Thompson y Duvall [1996] y McCarthy y Schneider [1995], pero es contrario al obtenido por Choi, Kwon y Lobo [2000]. 
Tabla 4. Relevancia valorativa de los activos intangibles reconocidos

Modelos:

$$
\begin{aligned}
& P_{i t}=\gamma_{0}+\gamma_{1} B V_{i t}+\gamma_{2} X_{i t}+\varepsilon_{i t} \\
& P_{i t}=\gamma_{0}+\gamma_{1} \operatorname{Tg} B V_{i t}+\gamma_{2} X_{i t}+\gamma_{3} \operatorname{Intg}_{i t}+\varepsilon_{i t} \\
& P_{i t}=\gamma_{0}+\gamma_{1} \operatorname{Tg} B V_{i t}+\gamma_{2} X_{i t}+\gamma_{3} \operatorname{Intg}_{i t}+\gamma_{4}\left(X_{i t} \cdot \operatorname{Intg}_{i t}\right)+\varepsilon_{i t}
\end{aligned}
$$

Para reducir los inconvenientes del denominado "efecto escala" todas las variables incluidas en la regresión se encuentran deflactadas por el precio de mercado de la empresa a principios del periodo t. Los modelos se estiman mediante la información recogida de las fuentes citadas en el apartado cuarto. El periodo muestral abarca los cierres fiscales de los años 1991 a 1999 , donde $\mathrm{P}_{\mathrm{it}}$ : valor de mercado de las acciones en el momento $\mathrm{t}$; $\mathrm{BV}_{\mathrm{it}}$ : valor contable de los fondos propios en el momento $\mathrm{t}$; $\mathrm{X}_{\mathrm{it}}$ : resultado contable del periodo $(\mathrm{t}-1, \mathrm{t}) ; \mathrm{TgBV}_{\mathrm{it}}$ : valor contable de los elementos tangibles en el periodo $\mathrm{t}$; $\operatorname{Intg}_{\mathrm{it}}$ : Activos intangibles reconocidos en el balance en el periodo $t ; R^{2}$ aj: Coeficiente de determinación ajustado de la regresión, N: Número de observaciones incluidas en la regresión

\begin{tabular}{ccccccccc}
\hline Modelo & Constante & $\mathbf{B V}$ & $\mathbf{X}$ & $\mathbf{T g B V}$ & $\mathbf{I n t g}$ & $\mathbf{( X \cdot I n t g )}$ & $\mathbf{R}^{2}$ aj. & $\mathbf{N}$ \\
\hline \multirow{2}{*}[2]{} & $0,9456^{* * *}$ & $0,1545^{* * *}$ & $1,8809^{* * *}$ & & & & 0,1608 & 787 \\
& $(21,24)$ & $(3,98)$ & $(6,89)$ & & & & & \\
{$[3]$} & $0,8916^{* * *}$ & & $2,0673^{* * *}$ & $0,1325^{* * *}$ & $0,9326^{* * *}$ & & 0,1758 & 787 \\
& $(20,74)$ & & $(7,22)$ & $(3,27)$ & $(4,90)$ & & & \\
{$[4]$} & $0,8897^{* * *}$ & & $2,1097^{* * *}$ & $0,1321^{* *}$ & $0,9520^{* * *}$ & $-0,6294$ & 0,1748 & 787 \\
& $(20,69)$ & $(4,78)$ & $(3,22)$ & $(3,95)$ & $(-0,13)$ & $0,178)$ \\
\hline
\end{tabular}

Entre paréntesis los t-estadísticos de significatividad individual basados en los errores estándar consistente ante heterocedasticidad de White [1980].

$* * *$ Significativo al $1 \% \quad * *$ Significativo al $5 \% \quad *$ Significativo al $10 \%$

Por último, nos gustaría señalar que se han repetido la estimación de los tres modelos mediante la utilización de variables sin deflactar, así como con la utilización de otros deflactores distintos al precio de mercado y que han sido propuestos en la literatura, como son el activo total y el valor contable de los fondos propios. Los resultados obtenidos son similares a los presentados ${ }^{6}$.

\subsection{Activos intangibles no reconocidos}

Tal y como se ha indicado en el apartado cuarto, tras la estimación del modelo [3], procedemos a medir los activos intangibles no reconocidos mediante la siguiente

${ }^{6}$ La significatividad de los coeficientes es exactamente igual a la indicada en la Tabla 1. No obstante, cabe señalar que se obtienen valores más altos tanto para el coeficiente del resultado contable (entre 3,38 y 5,08 ) como para el del valor contable de los fondos propios de los elementos tangibles (entre 0,23 y 0,31). En algún caso el término de interacción alcanza un valor positivo, pero en ningún caso es una variable significativa, ya que obtiene $\mathrm{p}$-valores superiores al $70 \%$. El $\mathrm{R}^{2}$ ajustado se encuentra entre 0,16 y 0,42 cuando se utilizan deflactores, mientras que en el caso de prescindir de ellos toma valores superiores a 0,90 , lo que corrobora la influencia del efecto escala mencionado en los trabajos que lo han estudiado. 
expresión, en la que los intangibles no reconocidos se aproximan por la diferencia entre el valor de mercado de la empresa y el valor dado por los inversores según las variables contables básicas y los activos intangibles que sí están recogidos en el balance:

$$
\operatorname{IntgNo} \operatorname{Re} c=P_{i t}-\left(2,0673 X_{i t}+0,1325 T g B V_{i t}+0,9326 \text { Intg }\right)
$$

La Tabla 5 muestra la evolución en el periodo muestral de la medida propuesta de activos intangibles no reconocidos. Merece la pena destacar como, en consonancia con lo observado en la evolución del ratio $\mathrm{B} / \mathrm{M}$, el nivel de intangibles no reconocidos en el balance ha aumentado de forma considerable en los últimos 3 años del periodo, lo que pone de manifiesto que en el periodo 1997-1999 los inversores han tenido en cuenta atributos de valor no reconocidos en contabilidad por un importe superior al $50 \%$ del activo, en términos de valores medianos.

\section{Tabla 5. Medición de los activos intangibles no reconocidos}

La siguiente tabla muestra la media, mediana y el número de observaciones en cada año del periodo muestral de los activos intangibles, calculado como el cociente entre los activos intangibles no reconocidos según la expresión [5] y el activo total.

\begin{tabular}{crrr}
\hline Año del cierre fiscal & Media & Mediana & Observaciones \\
\hline $\mathbf{1 9 9 1}$ & 0,3795 & 0,2653 & 89 \\
$\mathbf{1 9 9 2}$ & 0,3162 & 0,2423 & 92 \\
$\mathbf{1 9 9 3}$ & 0,4898 & 0,4430 & 94 \\
$\mathbf{1 9 9 4}$ & 0,4899 & 0,3971 & 86 \\
$\mathbf{1 9 9 5}$ & 0,4008 & 0,2916 & 86 \\
$\mathbf{1 9 9 6}$ & 0,4806 & 0,3886 & 81 \\
$\mathbf{1 9 9 7}$ & 0,8311 & 0,5732 & 82 \\
$\mathbf{1 9 9 8}$ & 0,9910 & 0,6835 & 92 \\
$\mathbf{1 9 9 9}$ & 0,7274 & 0,4988 & 85 \\
\hline Total & $\mathbf{0 , 5 6 6 2}$ & $\mathbf{0 , 4 1 3 0}$ & $\mathbf{7 8 7}$ \\
\hline
\end{tabular}

Y por último, al igual que en el apartado de análisis descriptivo, podemos intentar clasificar las empresas con mayores intangibles no reconocidos en función de las variables utilizadas en el análisis de rentabilidades. Concretamente, formamos 4 carteras en función del nivel de intangibles no reconocidos y calculamos los valores promedio de cada cartera del nivel de intangibles reconocidos, del ratio $\mathrm{B} / \mathrm{M}$, del error de predicción y seguimiento de los analistas financieros.

Los resultados se muestran en la Tabla 6, y nos indican que las empresas con mayor nivel de intangibles no reconocidos presentan un menor ratio $\mathrm{B} / \mathrm{M}$, y son más seguidas por los analistas financieros, que además cometen menores errores en sus 
predicciones. No obstante, debemos indicar que no se observa una tendencia clara en los errores de predicción de los analistas, y que no se encuentra una relación significativa entre la cantidad de intangibles reconocidos y no reconocidos.

Tabla 6. Ratio $B / M$, intangibles reconocidos según los cuartiles de activos intangibles no reconocidos.

INR: Activos intangibles no reconocidos, medida como el cociente entre los activos intangibles no reconocidos y el activo total; Int Rec.: Intangibles reconocidos, medidos como el cociente entre los activos intangibles reconocidos y activo total; B/M: Ratio book-to-market; Error Pred.: Error de predicción, calculado como el valor absoluto del cociente (resultado real-predicción)/predicción; Atención: número de analistas que hacen predicciones de resultados para cada empresa de la muestra a lo largo del año t. El número de analistas que siguen a una empresa a lo largo del año se ha calculado como la media mensual de analistas que han realizado alguna predicción de beneficios en algún mes del año estudiado, esto es, la suma del número de analistas que realizan predicciones cada mes dividido por doce.

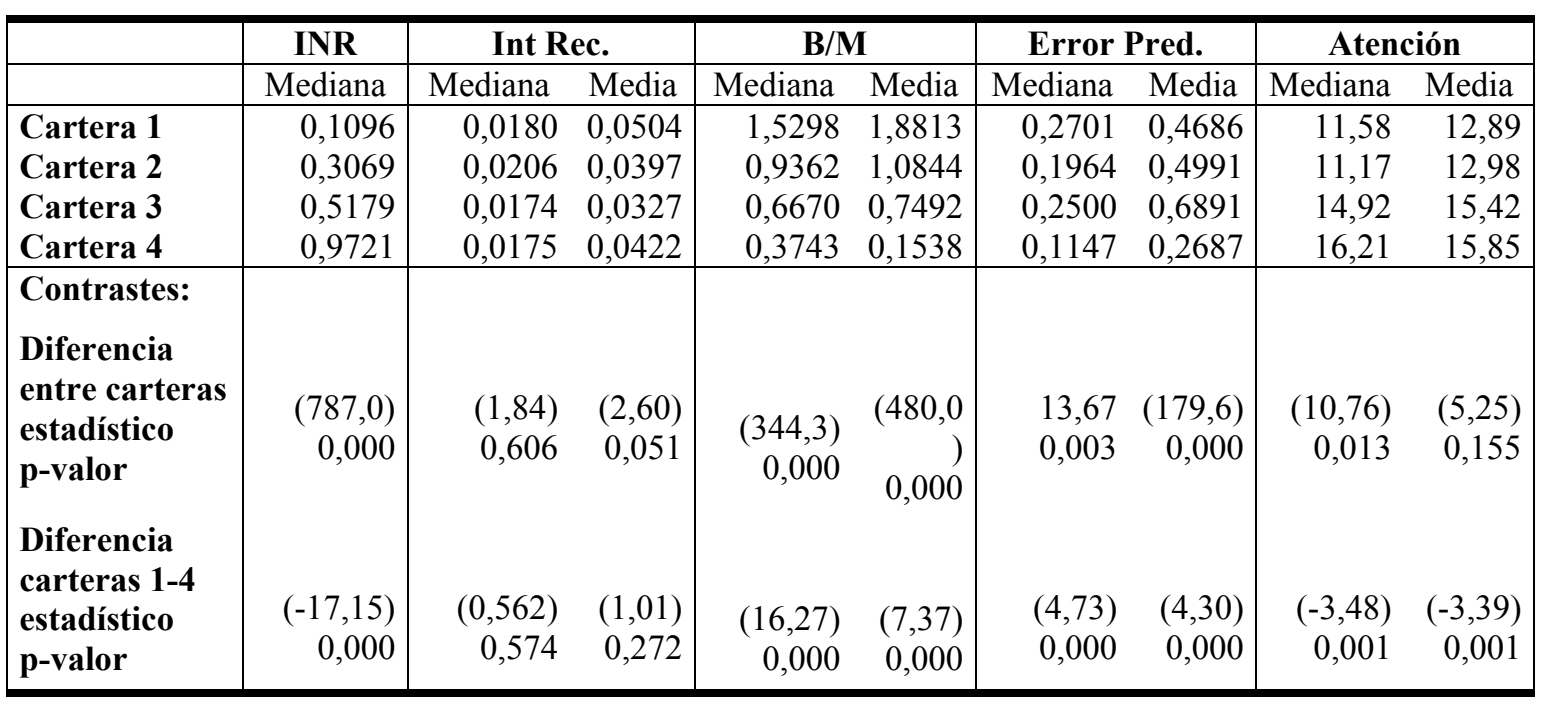

Los contrastes de diferencias de medias y medianas entre las cuatro carteras están basados en el estadístico F de un test Anova y en el estadístico chi-cuadrado del Test de Pearson, respectivamente.

Los contrastes de diferencias de medias y medianas entre las carteras 1 y 4 están basados en el estadístico $\mathrm{F}$ de un Test Anova y el test de rangos de Wilcoxon, respectivamente.

La explicación que damos a la evidencia obtenida es la siguiente. Las empresas con mayores intangibles no reconocidos deben presentar un ratio $\mathrm{B} / \mathrm{M}$ menor, pues los intangibles no reconocidos se han aproximado a través de la resta entre un valor de mercado y un valor contable estimado. Así, a mayor valor de mercado con relación al valor contable, mayor intangible no reconocido y menor $\mathrm{B} / \mathrm{M}$. Por otro lado, los analistas cometen menos errores de predicción en la cartera de mayores intangibles no reconocidos, lo que es consistente con la hipótesis de que los analistas prefieren que las empresas lleven los costes de intangibles directamente a resultados argumentada por Aboody y Lev [1998] para la industria del software en Estados Unidos, puesto que esto hace más fácil sus predicciones. Por último, no encontramos una explicación clara sobre 
el mayor seguimiento de las empresas con mayores niveles de intangibles no reconocidos, quizás esto pueda deberse a que es en estos casos donde mayor importancia adquiere la información privada y donde la labor del analista es más fácilmente repercutible al cliente.

Por último, una vez ya disponemos de todas las variables relevantes para implementar las estrategias de inversión, podemos calcular las correlaciones entre las mismas, tal y como se muestra en la Tabla 7. En la misma se observa, como cabía esperar, que el nivel de intangibles no reconocidos en balance y el ratio B/M tienen una correlación negativa y significativa al 1\%. También se observa que las empresas que tienen un mayor nivel de intangibles no reconocidos contablemente tienen un grado de seguimiento superior, así como que las empresas que reciben una mayor cobertura obtienen un menor error en la primera estimación realizada en el ejercicio.

Tabla 7. Coeficientes de correlación de Pearson entre las principales variables utilizadas para la formación de carteras

INR: Activos intangibles no reconocidos, medida como el cociente entre los activos intangibles no reconocidos y el activo total; B/M: Ratio book-to-market; Int. Rec.: Activos intangibles reconocidos contablemente, calculado como el cociente entre los activos intangibles reconocidos y el activo total; Error Pred.: Error de predicción calculado como el valor absoluto del cociente (resultado realpredicción)/predicción; Atención: número de analistas que hacen predicciones de resultados para cada empresa de la muestra a lo largo del año t, calculado como la media mensual de analistas que han realizado alguna predicción de beneficios en algún mes del año estudiado, esto es, la suma del número de analistas que realizan predicciones cada mes dividido por doce.

\begin{tabular}{lrrrr}
\hline & INR & B/M & Int. Rec. & Atención \\
\hline B/M & $-0,2564$ & & & \\
Int. Rec. & $(0,0000)$ & & & \\
& 0,0583 & $-0,0563$ & & \\
Atención & $(0,1019)$ & $(0,0877)$ & & \\
& 0,0922 & $-0,0475$ & $-0,0805$ & \\
Error Pred. & $(0,0097)$ & $(0,0937)$ & $(0,0118)$ & \\
& $-0,0391$ & 0,0705 & 0,0115 & $-0,0903$ \\
& $(0,2859)$ & $(0,0284)$ & $(0,7400)$ & $(0,0047)$ \\
\hline
\end{tabular}

p-valores del contraste de significatividad entre paréntesis

\section{Análisis de rentabilidades}

Como se ha indicado en el apartado de metodología, pretendemos analizar si el nivel de intangibles de las empresas, ya sean reconocidos o no contablemente, supone una mayor incertidumbre para el inversor, provocando que éste exija una rentabilidad en 
función del nivel de intangibles que posea una empresa. A su vez, se indagará las posibles interacciones existentes con los errores de predicción de los analistas, dada la posible desconfianza de los mismos a la capitalización de intangibles.

\subsection{Intangibles reconocidos contablemente y errores de predicción de los analistas}

\subsubsection{Errores de predicción en las estimaciones de beneficios de los analistas}

En primer lugar, y antes de entrar en la formación de carteras atendiendo a los niveles de intangibles y a los errores de predicción, creemos interesante comprobar si en el mercado español, los títulos cuyos errores de estimación han sido mayores en el año anterior, son percibidos como de mayor riesgo y exhiben, como consecuencia de esto, una mayor rentabilidad.

Para intentar averiguar esto, en el primer trimestre de cada ejercicio se ha obtenido el error producido para la primera estimación del consenso de los analistas que se realizó en el ejercicio anterior, de acuerdo con la información suministrada por $\mathrm{I} / \mathrm{B} / \mathrm{E} / \mathrm{S}$. Esto se realiza debido a que en este momento del tiempo es cuando se conoce la primera estimación de beneficios del presente ejercicio y el error producido para las del año anterior. Si un inversor estima que la incertidumbre de la estimación de consenso para el presente ejercicio depende del error que se produjo para la misma estimación en el ejercicio anterior, éste exigirá una rentabilidad en función del error producido para las estimaciones pasadas.

Procediendo con lo anterior, en el primer trimestre de cada ejercicio comprendido entre 1992 y 1999, los títulos disponibles son clasificados de menor a mayor en función del error de consenso, en magnitud absoluta, que se produjo para la primera estimación del ejercicio anterior. A continuación y de acuerdo con la clasificación previa, los títulos son asignados a diez carteras que representan los diferentes niveles de errores producidos en términos absolutos. Así, la cartera CE1 refleja el nivel de error más bajo y la cartera CE10 el más alto. Seguidamente, se calcula para cada cartera su rentabilidad en el presente ejercicio asignando idéntico peso a cada título que forma parte de la cartera. Actuando de esta forma, se dispone de una serie temporal de ocho rentabilidades anuales para cada cartera. 
En la Tabla 8 se ofrecen los resultados para cada una de las carteras. Como se puede observar en la segunda columna, tan sólo las rentabilidades de las carteras segunda, tercera y cuarta son significativas al $5 \%$ y positivas, aunque al realizar el test de Wald, se observa que se acepta la hipótesis de que la rentabilidad media para las distintas carteras construidas en función del error absoluto es la misma.

Una vez realizado este análisis se considera interesante tener en cuenta de forma explícita la existencia de diferentes niveles de riesgo. Para ello, se analiza si el comportamiento previamente observado persiste una vez se ajusta por riesgo las carteras en el contexto del CAPM, estimando la siguiente ecuación:

$$
R_{p t}-r_{f t}=\alpha_{p}+\beta_{p}\left(R_{M t}-r_{f t}\right)+e_{p t} \quad \begin{aligned}
& p=1,2, \ldots, 10 \\
& t=1,2, \ldots, 95
\end{aligned}
$$

donde $R_{p t}$ representa la rentabilidad de la cartera $\mathrm{p}$ en el mes t, $r_{f t}$ es la rentabilidad del activo libre de riesgo en el mes t, $\alpha_{p}$ es el alfa de Jensen y $R_{M t}$ representa la rentabilidad del índice de mercado equiponderado.

En la tercera columna de la Tabla 8 se verifica que los alfas de Jensen no son significativamente distintos de cero para ninguna cartera, aceptándose la hipótesis de ausencia de rentabilidad anormal para las diez carteras conjuntamente. Por tanto, en este contexto, se puede afirmar que el CAPM es capaz de explicar las rentabilidades obtenidas para las carteras clasificadas de acuerdo con el error de la primera estimación de consenso del ejercicio anterior. Por otra parte, es necesario comentar que no se observan mayores rentabilidades para las carteras que ostentan mayores errores de predicción, si bien aparecen como carteras más arriesgadas.

\subsubsection{Intangibles reconocidos contablemente}

Ely y Waymire (1999) muestran cómo la discrecionalidad en el reconocimiento de los activos intangibles no incrementa la claridad en la cantidad de información relevante para los inversores. Su estudio lo realizan sobre los datos contables estadounidenses disponibles antes del establecimiento de la SEC en 1930, época en la que existía discrecionalidad por parte de las empresas para reconocer sus activos intangibles. Estos autores afirman que, bajo estas condiciones, los gestores pueden sobreestimar los beneficios a través de la capitalización de los intangibles. 
Tabla 8. Resultados obtenidos teniendo en cuenta el error obtenido en las predicciones de beneficios de los analistas financieros

\begin{tabular}{cccc}
\hline & Rentabilidad Media & Alfas de Jensen & Beta \\
\hline \multirow{2}{*}{ CE1 } & 0,1393 & $-0,0481$ & $0,8639^{* * *}$ \\
& $(1,797)$ & $(-1,793)$ & $(7,259)$ \\
CE2 & $0,1857^{* *}$ & 0,0126 & $0,7516^{* * *}$ \\
& $(2,439)$ & $(0,239)$ & $(4,007)$ \\
CE3 & $0,2796^{* *}$ & 0,0686 & $1,0519^{* * * *}$ \\
& $(2,542)$ & $(0,992)$ & $(6,761)$ \\
CE4 & $0,2553^{* *}$ & 0,0534 & $\left(4800^{* * *}\right.$ \\
& $(2,989)$ & $(1,861)$ & $1,1670^{* * * *}$ \\
CE5 & 0,1837 & $-0,0418$ & $(7,830)$ \\
& $(1,782)$ & $(-1,688)$ & $1,1884^{* * *}$ \\
CE6 & 0,1608 & $-0,0674$ & $(6,200)$ \\
& $(1,318)$ & $(-1,778)$ & $1,1958^{* * *}$ \\
CE7 & 0,1744 & $-0,0547$ & $(8,323)$ \\
\hline \multirow{2}{*}{ CE8 } & $(1,689)$ & $(-1,598)$ & $1,3069^{* * *}$ \\
& $0,2600^{*}$ & 0,0169 & $(6,304)$ \\
\hline \multirow{2}{*}{ CE9 } & $(2,070)$ & $(0,370)$ & $1,1824^{* * * *}$ \\
& 0,2266 & $-0,0008$ & $(5,675)$ \\
\hline CE10 & $(1,678)$ & $(-0,012)$ & $1,5170^{* * *}$ \\
& $0,2917^{* *}$ & 0,0221 & $(12,26)$ \\
\hline \multirow{2}{*}{ CE1-CE10 } & $(1,965)$ & $(0,372)$ & $-0,6531^{* * *}$ \\
& $-0,1524$ & $-0,0702$ & $(-3,948)$ \\
\hline \multirow{2}{*}{ test conjunto $\chi^{2}$} & $(-1,432)$ & $(-0,875)$ & \\
p-valor & 1,5119 & 4,8543 & \\
\hline
\end{tabular}

Estadísticos t entre paréntesis.

*** Significativo al $1 \% \quad * *$ Significativo al $5 \% \quad *$ Significativo al $10 \%$

Resulta una cuestión interesante el comprobar si bajo otras condiciones como las existentes en el mercado español para el período analizado (1991-1999), es decir, con una normativa contable estricta en el reconocimiento de los activos intangibles, el inversor puede percibir también que los beneficios puedan estar sobreestimados en función de los activos intangibles reconocidos contablemente, afectando al riesgo y por tanto, a la rentabilidad exigida por parte de éste. Si esto es así, las carteras con mayor nivel de intangibles deberían ofrecer mayores rentabilidades.

Para ello, al principio de cada ejercicio comprendido entre 1992 y 1999, los títulos disponibles son clasificados de menor a mayor en función del porcentaje de activos intangibles sobre el activo total recogido del balance consolidado del ejercicio anterior. A continuación y de acuerdo con la clasificación previa, los títulos son asignados a diez carteras que representan los diferentes niveles de intangibles reconocidos contablemente en el balance consolidado. Así, la cartera CI1 refleja el porcentaje de intangibles más bajo y la cartera CI10 el más alto. Seguidamente, se 
calcula para cada cartera su rentabilidad en el presente ejercicio asignando idéntico peso a cada título que forma parte de la cartera. Actuando de esta forma, se dispone de una serie temporal de ocho rentabilidades anuales para cada cartera.

Los resultados se ofrecen en la Tabla 9, observando en la segunda columna que la rentabilidad no crece en función del porcentaje de intangibles reconocidos. Tan solo las rentabilidades de la sexta, octava y décima cartera no son significativas al 10\%, el resto son significativamente positivas. Se acepta la hipótesis de que las rentabilidades medias de las diferentes carteras sea la misma. Una vez se ajusta por riesgo, no se observa ningún alfa de Jensen significativamente distinto de cero con un nivel de significación del 5\%, aceptándose la hipótesis de ausencia de rentabilidad anormal para las diez carteras conjuntamente.

Tabla 9. Resultados obtenidos según el nivel de intangibles reconocidos

\begin{tabular}{|c|c|c|c|}
\hline & Rentabilidad Media & Alfas de Jensen & Beta \\
\hline CI1 & $\begin{array}{l}0,1484^{*} \\
(2,292)\end{array}$ & $\begin{array}{l}-0,0021 \\
(-0,053)\end{array}$ & $\begin{array}{c}0,5719^{\text {*** }} \\
(5,153)\end{array}$ \\
\hline $\mathrm{CI} 2$ & $\begin{array}{l}0,2767^{*} \\
(2,340)\end{array}$ & $\begin{array}{c}0,0228 \\
(0,390)\end{array}$ & $\begin{array}{c}0,3928^{* * *} \\
(6,795)\end{array}$ \\
\hline $\mathrm{CI} 3$ & $\begin{array}{l}0,1753^{*} \\
(1,899)\end{array}$ & $\begin{array}{l}-0,0115 \\
(-0,241)\end{array}$ & $\begin{array}{c}0,8597^{* 3 *} \\
(3,756)\end{array}$ \\
\hline CI4 & $\begin{array}{l}0,2110^{*} \\
(2,254)\end{array}$ & $\begin{array}{l}0,0073 \\
(0,197)\end{array}$ & $\begin{array}{r}0,9936^{* * * *} \\
(7,498)\end{array}$ \\
\hline CI5 & $\begin{array}{l}0,3643^{*} \\
(2,159)\end{array}$ & $\begin{array}{c}0,1502 \\
(1,131)\end{array}$ & $\begin{array}{r}1,0768^{* * * *} \\
(5,363)\end{array}$ \\
\hline CI6 & $\begin{array}{c}0,1974 \\
(1,752)\end{array}$ & $\begin{array}{l}-0,0410 \\
(-1,114)\end{array}$ & $\begin{array}{c}1,2698^{* * * *} \\
(7,763)\end{array}$ \\
\hline CI7 & $\begin{array}{l}0,2411^{* *} \\
(2,448)\end{array}$ & $\begin{array}{l}0,0348 \\
(0,677)\end{array}$ & $\begin{array}{l}1,0146^{* * *} \\
(8,175)\end{array}$ \\
\hline CI8 & $\begin{array}{l}0,1905 \\
(1,690)\end{array}$ & $\begin{array}{l}-0,0385 \\
(-1,268)\end{array}$ & $\begin{array}{l}1,1950 \\
(10,275)\end{array}$ \\
\hline CI9 & $\begin{array}{l}0,2896 \\
(2,219)\end{array}$ & $\begin{array}{l}0,0556 \\
(0,989)\end{array}$ & $\begin{array}{c}1,2347^{* * * 6} \\
(4,838)\end{array}$ \\
\hline CI10 & $\begin{array}{l}0,1263 \\
(1,082)\end{array}$ & $\begin{array}{l}-0,0906^{*} \\
(-2,183)\end{array}$ & $\begin{array}{c}1,0994^{* \cdots *} \\
(6,906)\end{array}$ \\
\hline CI1-CI10 & $\begin{array}{l}0,0220 \\
(0,262) \\
\end{array}$ & $\begin{array}{l}0,0885 \\
(1,446) \\
\end{array}$ & $\begin{array}{l}-0,5275^{* *} \\
(-3,263) \\
\end{array}$ \\
\hline $\begin{array}{c}\text { test conjunto } \chi^{2} \\
\text { p-valor }\end{array}$ & $\begin{array}{l}2,4739 \\
0,9816\end{array}$ & $\begin{array}{l}4,7892 \\
0,8523\end{array}$ & \\
\hline
\end{tabular}

Estadísticos t entre paréntesis.

$* * *$ Significativo al $1 \% \quad * *$ Significativo al $5 \% \quad *$ Significativo al $10 \%$

Por tanto, los resultados obtenidos, no muestran diferentes rentabilidades en función del nivel de intangibles reconocido contablemente, con lo que se aporta 
evidencia de que, dado que la normativa contable española es restrictiva a la hora del reconocimiento de los activos intangibles, quizás el inversor no perciba cómo más arriesgado un título cuyo balance consolidado muestre un mayor porcentaje de activos intangibles sobre el activo total.

\subsubsection{Interacción entre el nivel de intangibles reconocidos contablemente y los errores de predicción}

Una vez observado que los diferentes niveles de intangibles reconocidos contablemente no proporcionan distintas rentabilidades, es conveniente intentar indagar si ocurre lo mismo cuando controlamos esto según el error cometido en las estimaciones de beneficios ${ }^{7}$, dada la evidencia obtenida por Lev y Aboody [1998].

Para lograr lo anterior, los datos son ordenados anualmente de acuerdo con el porcentaje de activos intangibles sobre el activo total que poseen en el balance consolidado y también con respecto a la magnitud en términos absolutos cometida para la primera estimación de consenso del ejercicio anterior. Así, los títulos disponibles se ordenan de acuerdo con su grado de intangibles en tres percentiles estando formado por los títulos de menor nivel de intangibles el primero de ellos. Simultáneamente, los títulos son ordenados independientemente de acuerdo con su error en tres percentiles siendo el primero de ellos el que representa a los títulos de menor error. A partir de estas dos clasificaciones se ordenan los títulos anualmente en nueve carteras, exigiéndole a cada título para que pueda formar parte de una de estas carteras que pertenezca simultáneamente al percentil requerido de intangibles y de error ${ }^{8}$. Seguidamente, se calcula la rentabilidad en el presente ejercicio de cada cartera. Actuando así, se obtienen una serie temporal de ocho rentabilidades anuales para cada cartera.

En el panel A de la Tabla 10 aparecen las rentabilidades medias para cada una de las nueve carteras, observándose como aspecto más relevante que las carteras que contienen un menor error de predicción, independientemente del nivel de intangibles, obtienen rentabilidades positivas y significativas al 10\%. También se obtienen

\footnotetext{
7 También se ha controlado por el grado de atención, obteniéndose idénticos resultados a los mostrados en este apartado. Los resultados serán facilitados a toda persona que esté interesada en los mismos.

${ }^{8}$ Con la finalidad de conseguir un nivel mínimo de diversificación en las carteras, a cada una de las nueve carteras se le exige que en cualquier momento del tiempo tenga al menos cinco títulos. Este mismo requisito se le exige a todas las carteras mostradas en este trabajo que han sido construidas con la misma metodología.
} 
rentabilidades positivas y significativas al $10 \%$ en las carteras de menor nivel de intangibles e intermedio de error y en la de grado intermedio de intangibles y mayor error. Por otra parte, no hay ninguna diferencia entre carteras extremas que sea significativa.

\section{Tabla 10. Rentabilidades obtenidas mediante el análisis conjunto del nivel de intangibles} (CI) y los errores de predicción (CEP)

*I: En esta fila se presentan las diferencias entre las carteras con el nivel inferior y superior de intangibles reconocidos, dado un nivel de error predictivo. El recuadro situado en la esquina derecha de esta fila representa la diferencia entre la cartera de intangibles reconocidos y error de predicción más bajo y la cartera con mayor nivel en estas dos características.

*EP: En esta columna se presentan las diferencias entre las carteras con el nivel inferior y superior de errores, dado un nivel de intangibles reconocidos.

PANEL A: Rentabilidades puras

\begin{tabular}{c|cccc}
\hline & CEP-1 & CEP-2 & CEP-3 & *EP \\
\hline \multirow{2}{*}{ CI-1 } & $0,1905^{* *}$ & $0,2358^{*}$ & 0,1974 & $-0,0069$ \\
& $(2,376)$ & $(2,284)$ & $(1,734)$ & $(-0,057)$ \\
\hline \multirow{2}{*}{ CI-2 } & $0,2204^{*}$ & 0,1739 & $0,2933^{* *}$ & $-0,0729$ \\
& $(2,286)$ & $(1,807)$ & $(2,419)$ & $(-1,444)$ \\
\hline \multirow{2}{*}{ CI-3 } & $0,1644^{*}$ & 0,1868 & 0,2678 & $-0,1034$ \\
& $(2,070)$ & $(1,762)$ & $(1,415)$ & $(-0,742)$ \\
\hline \multirow{2}{*}{ I } & 0,0261 & 0,0490 & $-0,0703$ & $-0,0772$ \\
& $(0,406)$ & $(0,578)$ & $(-0,771)$ & $(-0,414)$ \\
\hline
\end{tabular}

PANEL B: Rentabilidades ajustadas por riesgo

\begin{tabular}{c|cccc}
\hline & CEP-1 & CEP-2 & CEP-3 & *EP \\
\hline \multirow{2}{*}{ CI-1 } & 0,0208 & 0,0159 & $-0,0061$ & 0,0268 \\
& $(0,465)$ & $(0,364)$ & $(-0,080)$ & $(0,2339)$ \\
\hline \multirow{2}{*}{ CI-2 } & 0,0079 & $-0,0501^{*}$ & 0,0630 & $-0,0550$ \\
& $(0,249)$ & $(-2,291)$ & $(1,234)$ & $(-1,141)$ \\
\hline \multirow{2}{*}{ CI-3 } & $-0,0113$ & $-0,0353$ & $-0,0246$ & 0,0133 \\
& $(-0,209)$ & $(-1,467)$ & $(-0,527)$ & $(0,192)$ \\
\hline \multirow{2}{*}{ I } & 0,0321 & 0,0512 & 0,0186 & 0,0454 \\
& $(0,576)$ & $(0,767)$ & $(0,353)$ & $(0,530)$ \\
\hline
\end{tabular}

Estadísticos t entre paréntesis

*** Significativo al 1\% * * Significativo al 5\% * * Significativo al 10\%

Antes de exponer las conclusiones, es necesario replicar el análisis anterior teniendo en cuenta el efecto que el riesgo tiene sobre la rentabilidad. Para ello, se emplean rentabilidades ajustadas por riesgo obtenidas a partir del CAPM, mostrando los resultados en el panel B de la Tabla 10.

Se puede observar que ninguna cartera obtiene rentabilidades anormales significativas al 5\%, una vez se ajusta por riesgo, tampoco ninguna diferencia entre carteras obtiene rentabilidades anormales significativas. Por todo lo anterior, se puede 
afirmar que no se observan rentabilidades diferentes según el nivel de intangibles de los activos financieros, ni tan siquiera, cuando tenemos en cuenta su posible interacción con el diferente nivel de riesgo de estimación que pueden tener los títulos que cotizan en el mercado español de capitales.

\subsection{Intangibles no reconocidos contablemente y errores de predicción de los analistas}

\subsubsection{Intangibles no reconocidos contablemente}

Una vez observado que los distintos niveles de activos intangibles reconocidos contablemente que poseen las empresas no afectan de manera diferente a la rentabilidad, sería oportuno investigar si ocurre lo mismo cuando se trata de aquellos activos intangibles que no son reconocidos en la contabilidad, pero sí son tenidos en cuenta por el mercado de capitales.

Como subrogado de los activos intangibles sin reconocimiento contable se ha utilizado la expresión [5], tal y como se expuso anteriormente. Esta medida representa en gran parte, el valor que atribuye el inversor a los activos intangibles sin reflejo contable, por lo que se puede pensar que cuanto mayor valoración haya dado el mercado a este tipo de activos, el inversor exigirá una mayor rentabilidad, ya que, en principio la incertidumbre sobre estos activos es mayor.

Para intentar averiguar lo expuesto anteriormente, al principio de cada ejercicio comprendido entre 1992 y 1999, los títulos disponibles son clasificados de menor a mayor en función del valor del porcentaje de activos intangibles no reconocidos contablemente. A continuación y de acuerdo con la clasificación previa, los títulos son asignados a diez carteras que representan los diferentes niveles de intangibles no reconocidos contablemente. Así, la cartera CINR1 refleja el porcentaje de intangibles no reconocido más bajo y la cartera CINR10 el más alto. Seguidamente, se calcula para cada cartera su rentabilidad en el presente ejercicio asignando idéntico peso a cada título que forma parte de la cartera. Actuando de esta forma, se dispone de una serie temporal de ocho rentabilidades anuales para cada cartera.

Los resultados obtenidos se muestran en la Tabla 11, observando en la segunda columna que la rentabilidad crece monótonamente conforme aumenta el nivel de intangibles no reconocidos contablemente, ofreciendo la cartera extrema superior una rentabilidad anual cercana al $105 \%$. La diferencia entre las carteras extremas inferior y 
superior es significativa con un nivel de significación inferior al $1 \%$ y a favor de la cartera superior en un $117 \%$ de rentabilidad anual. Indudablemente, se rechaza al 1\% la hipótesis nula de que la rentabilidad media sea la misma para todas las carteras. En este contexto adquiere una gran importancia el realizar el análisis anterior teniendo en cuenta de forma explícita el riesgo, para lo cual se realiza un ajuste por riesgo con el CAPM, mostrándose los resultados en las columnas tercera y cuarta de esta misma tabla.

Tabla 11. Resultados obtenidos teniendo en cuenta el grado de intangibles no reconocidos

\begin{tabular}{|c|c|c|c|}
\hline & Rentabilidad Media & Alfas de Jensen & Beta \\
\hline CINR1 & $\begin{array}{l}-0,1218 \\
(-1,552)\end{array}$ & $\begin{array}{c}-0,2891^{* * * *} \\
(-7,241)\end{array}$ & $\begin{array}{c}0,7048^{* * 3} \\
(3,490)\end{array}$ \\
\hline CINR2 & $\begin{array}{l}-0,0991 \\
(-1,549)\end{array}$ & $\begin{array}{l}-0,2674^{* * *} \\
(-10,453)\end{array}$ & $\begin{array}{c}0,7132^{* *} \\
(9,442)\end{array}$ \\
\hline CINR3 & $\begin{array}{l}-0,0645 \\
(-0,826)\end{array}$ & $\begin{array}{l}-0,2593^{* * *} \\
(-14,482)\end{array}$ & $\begin{array}{l}0,9235^{* *} \\
(16,438)\end{array}$ \\
\hline CINR4 & $\begin{array}{l}0,0256 \\
(0,263)\end{array}$ & $\begin{array}{c}-0,1755^{* * *} \\
(-5,644)\end{array}$ & $\begin{array}{r}0,9729^{* * *} \\
(7,587)\end{array}$ \\
\hline CINR5 & $\begin{array}{l}0,0717 \\
(0,815)\end{array}$ & $\begin{array}{c}-0,1306^{* * *} \\
(-5,875)\end{array}$ & $\begin{array}{l}0,9834^{* * *} \\
(13,591)\end{array}$ \\
\hline CINR6 & $\begin{array}{l}0,2192^{* *} \\
(2,616)\end{array}$ & $\begin{array}{l}0,0251 \\
(0,858)\end{array}$ & $\begin{array}{l}0,9176 \\
(24,438)\end{array}$ \\
\hline CINR7 & $\begin{array}{c}0,2774^{* *} \\
(2,792)\end{array}$ & $\begin{array}{c}0,0505 \\
(1,615)\end{array}$ & $\begin{array}{l}1,1777^{* * *} \\
(13,675)\end{array}$ \\
\hline CINR8 & $\begin{array}{l}0,3625^{* *} \\
(3,348)\end{array}$ & $\begin{array}{c}0,1310^{* * *} \\
(3,739)\end{array}$ & $\begin{array}{r}1,2146 \\
(7,501)\end{array}$ \\
\hline CINR9 & $\begin{array}{c}0,5364^{* * *} \\
(3,901)\end{array}$ & $\begin{array}{c}0,2588^{* * *} \\
(6,981)\end{array}$ & $\begin{array}{l}1,5813^{\text {*** }} \\
(18,990)\end{array}$ \\
\hline CINR10 & $\begin{array}{c}1,0497^{* * * *} \\
(5,271)\end{array}$ & $\begin{array}{c}0,7064 \\
(14,780)\end{array}$ & $\begin{array}{l}2,1026 \\
(11,921)\end{array}$ \\
\hline CINR1-CINR10 & $\begin{array}{c}-1,1715^{* * *} \\
(-7,366) \\
\end{array}$ & $\begin{array}{l}-0,9955^{* * *} \\
(-23,046) \\
\end{array}$ & $\begin{array}{r}-1,3978^{* *} \\
(-4,669) \\
\end{array}$ \\
\hline $\begin{array}{c}\text { test conjunto } \chi^{2} \\
\text { p-valor }\end{array}$ & $\begin{array}{c}45,189 \\
0,000001\end{array}$ & $\begin{array}{c}407,34 \\
0,000000\end{array}$ & \\
\hline
\end{tabular}

Estadísticos t entre paréntesis.

$* * *$ Significativo al $1 \% \quad * *$ Significativo al $5 \% \quad *$ Significativo al $10 \%$

Como se observa en la tercera columna, la rentabilidad anormal crece monótonamente conforme aumenta el nivel de intangibles sin reconocimiento en la contabilidad empresarial, siendo la diferencia entre las carteras extremas próxima al $100 \%$ y significativa al 1\%. Por otra parte, se rechaza la hipótesis nula de ausencia de rentabilidad anormal para las diez carteras conjuntamente con un nivel de significación inferior al $1 \%$.

Estos resultados muestran que los inversores obtuvieron para el período analizado una mayor rentabilidad ajustada al riesgo para aquellas carteras cuya 
valoración de los activos intangibles sin reflejo en el balance consolidado era mayor, por tanto se puede afirmar que el CAPM sería incapaz de explicar el diferencial de rentabilidad existente en las carteras clasificadas de acuerdo con su nivel de intangibles no reconocidos contablemente. De esta forma, queda patente que los intangibles que son valorados por el mercado y no tienen reflejo contable, afectan al proceso de valoración de activos. Esto es debido, en parte, al diferente riesgo que puede percibir el mercado de capitales español según el nivel de intangibles sin reconocimiento contable que posea una empresa.

\subsubsection{Interacción entre los intangibles no reconocidos contablemente y los errores de predicción}

Los resultados obtenidos en el apartado anterior han mostrado que los diferentes niveles de intangibles sin reconocimiento contable suponen distintas rentabilidades, incluso después del ajuste por riesgo. Ahora se considera importante investigar si la rentabilidad se ve afectada de manera diferente una vez se controla por el nivel de error en la estimación 9 .

Para lograr lo anterior, los datos son ordenados anualmente de acuerdo con el nivel de activos intangibles no reconocidos contablemente y también con respecto a la magnitud en términos absolutos cometida para la primera estimación de consenso del ejercicio anterior. Así, los títulos disponibles se ordenan de acuerdo con su grado de intangibles en tres percentiles estando formado por los títulos de menor nivel de intangibles sin reconocimiento el primero de ellos. Simultáneamente, los títulos son ordenados independientemente de acuerdo con su error en tres percentiles siendo el primero de ellos el que representa a los títulos de menor error. A partir de estas dos clasificaciones se ordenan los títulos anualmente en nueve carteras, exigiéndole a cada título para que pueda formar parte de una de estas carteras que pertenezca simultáneamente al percentil requerido de intangibles y de error. Seguidamente, se calcula la rentabilidad en el presente ejercicio de cada cartera. Actuando así, se obtienen una serie temporal de ocho rentabilidades anuales para cada cartera.

En el panel A de la Tabla 12 se muestran las rentabilidades medias para cada una de las nueve carteras, observándose claramente cómo las rentabilidades de las carteras

\footnotetext{
9 También se ha controlado por el grado de atención, obteniéndose idénticos resultados a los mostrados en este apartado. Los resultados serán facilitados a toda persona que esté interesada en los mismos.
} 
con mayor grado de intangibles sin reconocimiento contable obtienen mayores rentabilidades, por lo que en principio se puede hablar de un efecto "intangibles no reconocidos". También se observa que la diferencia entre la cartera con menor grado de intangibles no reconocidos y menor error de predicción con respecto a la cartera con mayor nivel en ambas características es cercana al 70\% siendo significativa al 5\%.

Tabla 12. Rentabilidades obtenidas mediante el análisis conjunto de los intangibles no reconocidos (CINR) y los errores de predicción (CEP)

*INR: En esta columna se presentan las diferencias entre las carteras con el nivel inferior y superior de intangibles no reconocidos contablemente, dado un nivel de error predictivo. El recuadro situado en la parte inferior de esta columna representa la diferencia entre la cartera de intangibles no reconocidos y error de predicción más bajo y la cartera con mayor nivel en estas dos características.

*EP: En esta fila se presentan las diferencias entre las carteras con el nivel inferior y superior de errores, dado un nivel de intangibles no reconocidos.

PANEL A: Rentabilidades puras

\begin{tabular}{c|cccc}
\hline & CINR-1 & CINR-2 & CINR-3 & *INR \\
\hline \multirow{2}{*}{ CEP-1 } & $-0,0281$ & $0,1935^{* *}$ & $0,6447^{* * *}$ & $-0,6728^{* * *}$ \\
& $(-0.359)$ & $(2,454)$ & $(3,856)$ & $(-3,812)$ \\
\hline \multirow{2}{*}{ CEP-2 } & $-0,0471$ & 0,1542 & $0,6010^{* * *}$ & $-0,6481^{* * *}$ \\
& $(-0,571)$ & $(1,764)$ & $(3,939)$ & $(-5,882)$ \\
\hline \multirow{2}{*}{ CEP-3 } & $-0,1850^{* *}$ & 0,1064 & $0,6681^{* * *}$ & $-0,8530^{* * *}$ \\
& $(-0,809)$ & $(0,949)$ & $(3,688)$ & $(-7,030)$ \\
\hline \multirow{2}{*}{$*$ EP } & 0,1569 & 0,0871 & $-0,0234$ & $-0,6961^{* *}$ \\
& $(1,415)$ & $(1,510)$ & $(-0,112)$ & $(-3,250)$ \\
\hline
\end{tabular}

PANEL B: Rentabilidades ajustadas por riesgo

\begin{tabular}{c|cccc}
\hline & CINR-1 & CINR-2 & CINR-3 & ${ }^{*}$ INR \\
\hline \multirow{2}{*}{ CEP-1 } & $-0,1382$ & $-0,0002$ & $0,4510^{* * *}$ & $-0,5892^{* *}$ \\
& $(-1,345)$ & $(-0,009)$ & $(3,020)$ & $(-2,978)$ \\
\hline \multirow{2}{*}{ CEP-2 } & $-0,2317^{* * *}$ & $-0,0543^{* * *}$ & $0,2964^{* * *}$ & $-0,5281^{* * * *}$ \\
& $(-6,484)$ & $(-4,083)$ & $(4,661)$ & $(-5,885)$ \\
\hline \multirow{2}{*}{ CEP-3 } & $-0,3422$ & $-0,1133^{*}$ & $0,3605^{* * *}$ & $-0,7028^{* * *}$ \\
& $(-9,852)$ & $(-2,416)$ & $(6,680)$ & $(-22,182)$ \\
\hline \multirow{2}{*}{$*$ EP } & 0,2041 & $0,1131^{*}$ & 0,0905 & $-0,4987^{* *}$ \\
& $(1,504)$ & $(2,142)$ & $(0,578)$ & $(-3,322)$ \\
\hline
\end{tabular}

Estadísticos t entre paréntesis

$* * *$ Significativo al $1 \% \quad * *$ Significativo al $5 \% \quad *$ Significativo al $10 \%$

De nuevo la evidencia previa debe matizarse, dado que el análisis anterior se ha efectuado sin tener en cuenta el riesgo de forma explícita. Para solventar esto, se replica el análisis anterior utilizando rentabilidades ajustadas por riesgo obtenidas a partir del CAPM, mostrando los resultados en el panel B de la Tabla 12. Los resultados obtenidos al ajustar por riesgo coinciden con los obtenidos en la Tabla 11. Las carteras con mayor nivel de intangibles no reconocidos contablemente obtienen rentabilidades anormales 
positivas y significativas, siendo las diferencias entre los extremos también significativas al 5\%. Esta tabla aporta evidencia de un efecto "intangibles no reconocidos" incluso después de controlar por el nivel de error predictivo y de ajustar por riesgo, por lo que se puede afirmar que en el período analizado, las empresas con mayor nivel de intangibles no reflejados en balance, obtuvieron mayores rentabilidades anormales.

\subsection{Ratio book-to-market (B/M)}

Puesto que en la Tabla 7 se ha observado la correlación existente entre el Bookto-Market y los intangibles sin reconocimiento contable, parece interesante investigar si se obtienen resultados similares al utilizar como proxy de los intangibles no reconocidos el mencionado ratio.

En primer lugar, se analizará si en el mercado español en el período comprendido entre enero de 1992 y diciembre de 1999, se obtienen rentabilidades mayores para aquellas empresas cuyo ratio $\mathrm{B} / \mathrm{M}$ es menor. Puesto que el valor en libros de una sociedad representa el patrimonio contable propiedad de los accionistas, sería lógico pensar que las empresas que tengan un menor valor en el ratio analizado, suponen un mayor riesgo para el accionista. Por este motivo, se considera interesante observar si en el mercado español se produce este comportamiento.

Para ello, al final de cada mes del período muestral los títulos disponibles son clasificados de menor a mayor en función de su ratio $\mathrm{B} / \mathrm{M}$. A continuación, y de acuerdo con la clasificación previa, los títulos son asignados a diez carteras que representan cuál es la relación entre su valor contable y el de mercado. Así, la cartera CB1 refleja el ratio B/M más bajo y la cartera CB10 el más alto. Seguidamente, se calcula para cada cartera su rentabilidad en el mes siguiente al de formación asignando idéntico peso a cada título que forma parte de la cartera. Actuando de esta forma se dispone de una serie temporal de 95 rentabilidades mensuales para cada cartera y, adicionalmente, como consecuencia de la metodología de formación de cartera empleada se minimiza al máximo la posible aparición de un sesgo de supervivencia.

En la Tabla 13 se ofrecen los resultados para cada una de las carteras. Como se puede observar en la segunda columna, hay tres carteras (primera, sexta y novena) con rentabilidades positivas y significativas al 5\%, pero sin embargo el test de Wald acepta la hipótesis de que la rentabilidad media para las distintas carteras de B/M sea la misma. 
De nuevo, como se ha ido realizando a lo largo de este trabajo se procede a analizar los resultados mediante un ajuste por riesgo con el CAPM. Como se puede observar en la tercera columna de la Tabla 13, no se observa ninguna rentabilidad anormal significativamente distinta de cero con un nivel de significación del $5 \%$ y se acepta la hipótesis nula de ausencia de rentabilidad anormal para las diez carteras de B/M conjuntamente. Por tanto, los resultados obtenidos muestran que el CAPM sería capaz de explicar los diferentes niveles de rentabilidad de las carteras formadas en función del ratio analizado, no observándose ningún comportamiento anómalo.

Tabla 13. Resultados obtenidos teniendo en cuenta el ratio $\mathrm{B} / \mathrm{M}$

\begin{tabular}{|c|c|c|c|}
\hline & Rentabilidad Media & Alfas de Jensen & Beta \\
\hline CB1 & $\begin{array}{c}0,0188^{* *} \\
(2,152)\end{array}$ & $\begin{array}{l}0,0037 \\
(0,855)\end{array}$ & $\begin{array}{c}1,0744^{* * *} \\
(17,624)\end{array}$ \\
\hline CB2 & $\begin{array}{l}0,0120^{*} \\
(1,761)\end{array}$ & $\begin{array}{l}-0,0014 \\
(-0,568)\end{array}$ & $\begin{array}{c}0,8631^{* * * * *} \\
(11,885)\end{array}$ \\
\hline CB3 & $\begin{array}{l}0,0132^{*} \\
(1,899)\end{array}$ & $\begin{array}{l}-0,0009 \\
(-0,401)\end{array}$ & $\begin{array}{l}0,9530^{* * *} \\
(15,548)\end{array}$ \\
\hline CB4 & $\begin{array}{l}0,0109^{*} \\
(1,661)\end{array}$ & $\begin{array}{l}-0,0030 \\
(-1,591)\end{array}$ & $\begin{array}{l}0,9363^{* * *} \\
(24,416)\end{array}$ \\
\hline CB5 & $\begin{array}{l}0,0152^{*} \\
(1,977)\end{array}$ & $\begin{array}{l}0,0010 \\
(0,394)\end{array}$ & $\begin{array}{c}0,9761^{* * * *} \\
(16,542)\end{array}$ \\
\hline CB6 & $\begin{array}{l}0,0140^{* *} \\
(2,162)\end{array}$ & $\begin{array}{l}0,0001 \\
(0,060)\end{array}$ & $\begin{array}{l}0,9231^{* * *} \\
(16,769)\end{array}$ \\
\hline CB7 & $\begin{array}{l}0,0130^{*} \\
(1.681)\end{array}$ & $\begin{array}{l}-0,0010 \\
(-0,405)\end{array}$ & $\begin{array}{c}0,9484^{* * * *} \\
(15,843)\end{array}$ \\
\hline CB8 & $\begin{array}{l}0,0197^{*} \\
(1,947)\end{array}$ & $\begin{array}{l}0,0025 \\
(0,876)\end{array}$ & $\begin{array}{l}1,3314^{* * * *} \\
(14,639)\end{array}$ \\
\hline CB9 & $\begin{array}{l}0,0169^{* *} \\
(2,075)\end{array}$ & $\begin{array}{l}0,0016 \\
(0,551)\end{array}$ & $\begin{array}{l}1,0967^{* * * 3} \\
(17,143)\end{array}$ \\
\hline CB10 & $\begin{array}{l}0,0121 \\
(1,156)\end{array}$ & $\begin{array}{l}-0,0053 \\
(-1,328)\end{array}$ & $\begin{array}{c}1,3644^{* * * *} \\
(9,713)\end{array}$ \\
\hline CB1-CB10 & $\begin{array}{l}0,0067 \\
(1,010)\end{array}$ & $\begin{array}{l}0,0090 \\
(1,505)\end{array}$ & $\begin{array}{c}-0,2900^{* * *} \\
(-1,804)\end{array}$ \\
\hline $\begin{array}{c}\text { test conjunto } \chi^{2} \\
\text { p-valor }\end{array}$ & $\begin{array}{l}9,0562 \\
0,4321\end{array}$ & $\begin{array}{l}11,539 \\
0,3171\end{array}$ & \\
\hline
\end{tabular}

Estadísticos t entre paréntesis. ***Significativo al 1\% **Significativo al 5\% * Significativo al $10 \%$

A pesar de la correlación existente entre las dos aproximaciones utilizadas del valor de los intangibles que no aparecen en balance, es evidente que ambas no miden el mismo fenómeno, quizás propiciado en gran parte debido a que el ratio $\mathrm{B} / \mathrm{M}$ contiene información de los datos contables y de la valoración de mercado, no conteniendo explícitamente la parte del valor de mercado que no es extrapolable a partir de los datos contables. 


\subsubsection{Interacción entre el ratio $B / M$ y el grado de atención}

El resultado obtenido en el apartado anterior muestra la no existencia de rentabilidades anormales cuando se tiene en cuenta sólo el ratio $\mathrm{B} / \mathrm{M}$ de las empresas para conformar carteras de inversión. Una vez observado este resultado, puede surgir la cuestión de si este comportamiento persiste cuando se controla por el grado de atención que reciben las empresas en el mercado. Se trata de averiguar si teniendo en cuenta que las empresas pueden tener una diferente cobertura en función del ratio en estudio, esto puede descubrir diferentes rentabilidades al analizar conjuntamente estas dos características $^{10}$.

Puesto que el grado de atención que reciben las empresas ha sido aproximado por el número de estimaciones mensuales que realizan los analistas sobre las mismas, $\mathrm{y}$ se ha observado en la Tabla 7 que esta característica está correlacionada significativamente al $10 \%$ con el ratio $\mathrm{B} / \mathrm{M}$ de las compañías, se ha calculado el grado de atención independientemente del ratio en cuestión mediante la siguiente regresión en sección cruzada para cada uno de los meses en estudio:

$$
\log \left(1+\mathrm{Est}_{\mathrm{it}}\right)=\beta_{0}+\beta_{1}(\mathrm{~B} / \mathrm{M})_{\mathrm{it}}+\varepsilon_{\mathrm{it}} \quad \begin{gathered}
i=1,2, \ldots, n \\
t=1,2, \ldots, 95
\end{gathered}
$$

donde Est $_{\text {it }}$ representa el número de estimaciones recibidas para la compañía $\mathrm{i}$ en el mes t, $(\mathrm{B} / \mathrm{M})_{\text {it }}$ será el ratio book-to-market de la empresa i en el mes t, $\mathrm{n}$ representa el número de activos existentes en el mes t y $\varepsilon_{\text {it }}$, el grado de atención residual, es decir, independiente del ratio en estudio.

Una vez obtenido el grado de atención residual se construyen las carteras de acuerdo con las dos características. Los datos son ordenados mensualmente de acuerdo con el grado de atención residual y el ratio B/M. Así, los títulos disponibles se ordenan de acuerdo con su grado de atención residual en tres percentiles estando formado por los títulos de menor atención residual el primero de ellos. Simultáneamente, los títulos son ordenados independientemente de acuerdo con $\mathrm{su} \mathrm{B} / \mathrm{M}$ en tres percentiles siendo el primero de ellos el que representa a los títulos de menor ratio. A partir de estas dos

\footnotetext{
${ }^{10}$ Se ha controlado por el grado de atención, al disponer datos mensuales de esta variable al igual que del ratio $\mathrm{B} / \mathrm{M}$. Por otra parte con el fin de verificar los resultados, se ha investigado la interacción anual entre el mencionado ratio y los errores de predicción, obteniendo resultados similares a los mostrados en este apartado. Esto será facilitado a toda persona que lo solicite.
} 
clasificaciones se ordenan los títulos mensualmente en nueve carteras, exigiéndole a cada título para que pueda formar parte de una de estas carteras que pertenezca simultáneamente al percentil requerido de atención residual y al de B/M. Seguidamente, se calcula de igual forma que en los apartados anteriores la rentabilidad mensual de cada cartera. Actuando así, se obtienen nueve carteras que proporcionan una serie temporal ininterrumpida de 95 observaciones mensuales.

En el panel A de la Tabla 14 se muestran las rentabilidades medias para cada una de las nueve carteras construidas con el procedimiento descrito en el párrafo anterior. Se observa que en el nivel de atención residual superior, se obtienen rentabilidades positivas y significativas con un nivel de significación inferior al 10\%, siendo la diferencia entre la cartera con menor y mayor ratio B/M significativa también al mismo nivel y a favor de la cartera de mayor ratio. Esto, en principio, pone de manifiesto que las empresas con menor ratio, no obtuvieron mayores rentabilidades durante el período analizado. La cartera de menor atención residual y ratio obtiene una rentabilidad mensual cercana al $1,87 \%$ mensual y significativa al $5 \%$.

Antes de elaborar las conclusiones, es necesario replicar el análisis anterior teniendo en cuenta el efecto que el riego tiene sobre la rentabilidad. Para ello, se emplean rentabilidades ajustadas por riesgo obtenidas a partir del CAPM, mostrando los resultados en el panel B de la Tabla 14. Los resultados obtenidos muestran cómo una vez se ajusta por riesgo, tan sólo la rentabilidad de la cartera con mayor ratio y atención residual intermedia es significativa al 5\% y negativa.

Una vez observado los resultados conjuntos de la Tabla 14 se puede afirmar que el CAPM explicaría los diferentes niveles de rentabilidad ofrecidos por las carteras clasificadas de acuerdo con el ratio $\mathrm{B} / \mathrm{M}$, incluso cuando esta característica es controlada por el nivel de seguimiento, independiente del ratio en cuestión, que reciben los títulos. Por tanto, no se observa que en el mercado español los títulos con un menor $\mathrm{B} / \mathrm{M}$ hayan obtenido una mayor rentabilidad en el período analizado como consecuencia de soportar un mayor riesgo. 
Tabla 14. Rentabilidades obtenidas mediante el análisis conjunto del ratio B/M (CB)y el grado de atención residual (CAR)

*B: En esta fila se presentan las diferencias entre las carteras con el nivel inferior y superior del ratio $\mathrm{B} / \mathrm{M}$, dado un nivel de atención residual. El recuadro situado en la esquina derecha de esta fila representa la diferencia entre la cartera de atención residual y B/M más bajo y la cartera con mayor nivel en estas dos características.

*AR: En esta columna se presentan las diferencias entre las carteras con el nivel inferior y superior de atención residual, dado un nivel de ratio B/M.

PANEL A: Rentabilidades puras

\begin{tabular}{c|cccc}
\hline & CAR-1 & CAR-2 & CAR-3 & *AR \\
\hline \multirow{2}{*}{ CB-1 } & $0.0187^{* *}$ & $0.0144^{*}$ & $0.0132^{*}$ & 0.0055 \\
& $(2.251)$ & $(1.773)$ & $(1.924)$ & $(0.788)$ \\
\hline \multirow{2}{*}{ CB-2 } & 0.0099 & $0.0132^{*}$ & $0.0162^{* *}$ & -0.0064 \\
& $(1.497)$ & $(1.688)$ & $(2.172)$ & $(-1.385)$ \\
\hline \multirow{2}{*}{ CB-3 } & $0.0231^{*}$ & 0.0107 & $0.0196^{* *}$ & 0.0035 \\
& $(1.778)$ & $(1.271)$ & $(2.611)$ & $(0.408)$ \\
\hline \multirow{2}{*}{$*$ B } & -0.0044 & 0.0038 & $-0.0063^{*}$ & -0.0009 \\
& $(-0.520)$ & $(1.020)$ & $(-1.807)$ & $(-0.118)$ \\
\hline
\end{tabular}

PANEL B: Rentabilidades ajustadas por riesgo

\begin{tabular}{c|cccc}
\hline & CAR-1 & CAR-2 & CAR-3 & *AR \\
\hline \multirow{2}{*}{ CB-1 } & 0,0054 & $-0,0005$ & $-0,0007$ & 0,0061 \\
& $(1,076)$ & $(-0,202)$ & $(-0,227)$ & $(0,888)$ \\
\hline \multirow{2}{*}{ CB-2 } & $-0,0029$ & $-0,0015$ & 0,0017 & $-0,0046$ \\
& $(-1,054)$ & $(-0,688)$ & $(0,528)$ & $(-0,962)$ \\
\hline \multirow{2}{*}{ CB-3 } & 0,0039 & $-0,0053^{* *}$ & 0,0055 & $-0,0016$ \\
& $(0,794)$ & $(-2,164)$ & $(1,451)$ & $(-0,263)$ \\
\hline \multirow{2}{*}{$* \mathbf{B}$} & 0,0014 & 0,0048 & $-0,0062^{*}$ & $-0,0002$ \\
& $(0,225)$ & $(1,384)$ & $(-1,799)$ & $(-0,023)$ \\
\hline
\end{tabular}

Estadísticos $\mathrm{t}$ entre paréntesis

$* * *$ Significativo al $1 \% \quad * *$ Significativo al $5 \% \quad *$ Significativo al $10 \%$

\section{Conclusiones}

El reconocimiento contable de los activos intangibles ha sido una creciente preocupación de los principales organismos internacionales emisores de normas interesados en aumentar la utilidad de la información transmitida en los estados financieros. Una de las posibles causas de la pérdida de relevancia de las cuentas anuales puede encontrarse en el hecho de que no registrar contablemente activos intangibles que contribuyan de manera importante a la generación de ingresos de las empresas debilita la utilidad de la información. 
Observadas las restricciones existentes en el ámbito internacional para el reflejo en el balance de los activos intangibles, en este trabajo se indaga acerca de si en el mercado de capitales español las compañías han proporcionado diferentes niveles de rentabilidad en función de su grado de intangibles. Teniendo en cuenta que en nuestro país, la normativa que regula este tipo de activos es estricta en cuanto a las condiciones que permiten reconocerlos en el balance de situación, es una cuestión importante observar si los inversores perciben diferentes niveles de riesgo en función de los intangibles reflejados en el balance y como consecuencia de ello exigen diferentes rendimientos.

Los resultados obtenidos muestran que no existen diferentes niveles de rentabilidad en función del grado de intangibles reconocidos contablemente, lo que evidencia que quizás el inversor no percibe diferentes niveles de riesgo. Esto es en gran parte debido a que en España la regulación contable entorno a los activos intangibles es estricta durante todo el período analizado, por lo que el mercado de capitales percibe como legítimos los intangibles reflejados en el balance, no considerando que la capitalización de los intangibles se utilice para manipular el resultado empresarial. Este hallazgo puede estar indicando que hasta la actualidad la valoración contable de los activos intangibles no es percibida como una forma de alterar fraudulentamente el resultado empresarial.

Una vez obtenido el anterior resultado surge la cuestión de si existe una parte de activos intangibles sin posibilidad de ser registrados contablemente que el inversor valora como parte fundamental del patrimonio real de la empresa. Para ello se propone una medida de intangibles sin reconocimiento contable y se analiza la relación con la rentabilidad, comprobando que los diferentes niveles sí afectan a ésta. Se obtiene evidencia de que las empresas con mayor grado de intangibles no reconocidos, obtienen mayores rentabilidades, incluso después de ajustar por riesgo. Esto puede estar mostrando que la normativa española con respecto al reconocimiento de los intangibles al ser estricta, no es capaz de informar sobre esta importante fuente de valor que representa este tipo de activos para las compañías.

Por todo lo anterior, los hallazgos de este trabajo aportan evidencia acerca del distanciamiento existente en la actualidad entre el valor contable de los activos intangibles presentados en el balance de situación y el valor real de los mismos.

Por último, cabe mencionar que al construir la medida de intangibles no reconocidos contablemente utilizando una regresión de corte transversal no se reconoce la propia especificidad de las empresas, lo que aún suponiendo una limitación al 
presente trabajo no supone un impedimento para analizar los resultados obtenidos, puesto que esta medida tan sólo se utiliza para formar carteras en función del nivel estimado de intangibles sin reconocimiento en el balance. 


\section{REFERENCIAS BIBLIOGRÁFICAS}

ABOODY, D. [1996]: "Market Valuation of Employee Stock Options". Journal of Accounting and Economics, 22: 357-391.

ABOODY, D. Y LEV, B. [1998]: "The Value Relevance of Intangibles: The Case of Software Capitalization". Journal of Accounting Research, Supplement: 161-191.

AMIR, A.; HARRIS, T. S. Y VENUTI, E. K. [1993]: "A comparison of the Value-Relevance of US versus Non-US GAAP Accounting Measures Using 20-F Reconciliations". Journal of Accounting Research 31: 230-264.

AMIR, E. Y LEV, B. [1996]:"Value-Relevance of Nonfinancial Information: The Wireless Communications Industry". Journal of Accounting and Economics, 22: 3-30.

BARTH, M. Y CLINCH, G. [1998]: "Revalued Financial, Tangible, and Intangible Assets: Associations with Share Prices and Non-Market-Based Value Estimates". Journal of Accounting Research, Supplement: 199-233.

BARTH, M. E. Y KALLAPUR, S. [1996]: "The Effects of Cross-Sectional Scale Differences on Regressions Results in Empirical Accounting Research". Contemporary Accounting Research, Vol. 13, Fall: 527-567.

BARTH, M. E., BEAVER, W. H. Y LANDSMAN, W. R. [1998]: "Relative Valuation Roles of Equity Book Value and Net Income as a Function of Financial Health". Journal of Accounting and Economics, 25: 1-34.

BARTH, M.; ClEMENT, M.; FOSTER, G. Y KASZNIK, R. [1998]: "Brand Values and Capital Market Valuations". Review of Accounting Studies: 41-68.

BARTHOLDY, J. Y PEARE, P. [2001]: "The Relative Efficiency of Beta Estimates". Working Paper, Aarhus School of Business.

BLACK, E. L.; CARNES, T. A. Y VERNON, J.R. [1999]: "The market valuation of firm reputation". Working Paper. University of Arkansas and University of Kansas.

BROWN, S., LO, K. Y LYS, T. [1999]: "Use of R2 in Accounting Research: Measuring Changes in Value Relevance over the Last Four Decades". Journal of Accounting and Economics, Vol. 28, num. 2: 83-115.

BURGSTAHLER, D. C. Y DICHEV, I. D. [1997]: "Earnings, Adaptation and Equity Value". The Accounting Review, vol. 72, num. 2, April: 187-215.

CAÑIBANO, L.; GARCÍA-AYUSO, M Y SÁNCHEZ, M.P. [1999]: "La Relevancia de los Intangibles para la Valoración y la Gestión de Empresas: Revista Española de Financiación y Contabilidad, núm. 100: 17-88. 
CAÑIBANO, L.; GARCÍA-AYUSO, M Y SÁNCHEZ, M.P. [2000]: "La Valoración de los Intangibles: Estudios de Innovación vs Información Contable-Financiera" Análisis Financiero: 6-24.

CHAUVIN, K. W. Y HIRSCHEY, M. [1994]: "Goodwill, Profitability, and the Market Value of the Firm". Journal of Accounting and Public Policy 13: 15-180.

CHOI, W. W.; KWON, S. S Y LOBO, G. J. [2000]: "Market valuation of Intangible Assets". Journal of Business Research 49: 35-45.

COLLINS, D. W., MAYDEW, E. I. Y WEISS, IRA S. [1997]: "Changes in the ValueRelevance of Earnings and Book Values Over the Past Forty Years". Journal of Accounting and Economics, 24: 39-67.

EASTON, P. D. Y SOMMERS, G. [2000]: "Scale and Scale Effects in Market-Based Accounting Research". Working Paper, The Ohio State University.

ELY, K. Y WAYMIRE, G. [1999]: "Intangible Assets and Stock Prices in the Pre-SEC Era". Journal of Accounting Research, vol. 37, Supplement: 17-51.

EPSTEIN, L. G. Y TURNBULL, S. M. [1980]: "Capital Asset Prices and the Temporal Resolution of Uncertainty". Journal of Finance 35, June: 627-643.

FRANCIS, J. Y SCHIPPER, K. [1999]: "Have Financial Statement Lost Their Relevance?". Journal of Accounting Research, vol. 37, num. 2, Autumn: 319-352.

GOODWIN, J. [2002]: "Longitudinal Earnings Value Relevance and Intangible Assets: Evidence from Australian Firms, 1975-1999". Working paper. La Trobe University.

HOLLAND, J. [2001]: "Financial institutions, intangibles and corporate governance". Accounting, Auditing \& Accountability Journal, vol. 14, num. 4: 497-529.

INTERNATIONAL ACCOUNTING STANDARDS COMMITTEE [1998]: International Accounting Standard, num. 38, "Intangible Assets". London: IASC.

ITTNER, C. Y LARCKER, D. [1998]: "Are Nonfinancial Measures Leading Indicators of Financial Performance? An Analysis of Customer Satisfaction". Journal of Accounting Research, Supplement, 1-35.

JENNINGS, R.; ROBINSON, J.; THOMPSON II, R. B. Y DUVALL, L. [1996]: "The Relation between Accounting Goodwill Numbers and Equity Values". Journal of Business Finance and Accounting 23: 513-533.

KOHLBECK, M. Y WARFIELD, T. [2002]: "The Role of Unrecorded Intangible Assets in Residual Income Valuation: The Case of Banks". Working Paper. University of Wisconsin-Madison.

LARRÁN, M.; MONTERREY, J. Y MULERO, E. [2000]: "Una evaluación empírica del fondo de comercio". Revista de Contabilidad, Vol. 3, núm. 5: 101-126. 
LARRÁN, M.; REES, W. [1999]: "Propiedades de los pronósticos de Beneficios realizados por los Analistas Financieros: Una Aplicación al caso español". Revista Española de Financiación y Contabilidad, Vol. XXVIII, núm. 101: 675-729.

LEV, B. [2001]: Intangibles: Management, Measurement and Reporting (New York: Brookings Institution Press).

LEV, B. Y SOUGIANNIS, T. [1996]: "The Capitalization, Amortization, and Value-Relevance of R\&D". Journal of Accounting and Economics, February: 107-138

LEV, B. Y ZAROWIN, P. [1999]: "The Boundaries of Financial Reporting and How to Extend Them". Journal of Accounting Research, Autumn: 353-385.

LYS, T. Y SOO, L. [1995]: "Analysts' Forecast Precision as a Response to Competition". Journal of Accounting, Auditing and Finance 10: 751-765.

MCCARTHY, M. G. Y SCHNEIDER, D. K. [1995]: "Market Perception of Goodwill: Some Empirical Evidence". Accounting and Business Research 26: 69-81.

MERITUM PROJECT [2001]:"Guidelines for managing and reporting on intangibles (intellectual capital report)". June.

OHLSON, J. A. [1995]: "Earnings, Book Values, and Dividends in Equity Valuation". Contemporary Accounting Research, vol. 11, num. 2, Spring: 661-687.

STOLOWY, H. Y JENY-CAZAVAN, A. [2001]: "International accounting disharmony: the case of intangibles". Accounting, Auditing \& Accountability Journal, vol.14, num. 4: 477-496.

WHITE, H. [1980]: "A Heteroskedasciticy-Consistent Covariance Matrix Estimator and a Direct Test for Heteroskedasticity". Econometrica, May: 817-838.

WYATT, A. [2002]: "Towards a financial reporting framework for intangibles". Journal of Intellectual Capital, vol. 3, num. 1: 71-86. 